\title{
Expectation Invariants for Probabilistic Program Loops as Fixed Points
}

\author{
Aleksandar Chakarov*1 and Sriram Sankaranarayanan ${ }^{\dagger 1}$ \\ ${ }^{1}$ University of Colorado, Boulder, CO
}

October 9, 2014

\begin{abstract}
We present static analyses for probabilistic loops using expectation invariants. Probabilistic loops are imperative while-loops augmented with calls to random value generators. Whereas, traditional program analysis uses Floyd-Hoare style invariants to over-approximate the set of reachable states, our approach synthesizes invariant inequalities involving the expected values of program expressions at the loop head. We first define the notion of expectation invariants, and demonstrate their usefulness in analyzing probabilistic program loops. Next, we present the set of expectation invariants for a loop as a fixed point of the pre-expectation operator over sets of program expressions. Finally, we use existing concepts from abstract interpretation theory to present an iterative analysis that synthesizes expectation invariants for probabilistic program loops. We show how the standard polyhedral abstract domain can be used to synthesize expectation invariants for probabilistic programs, and demonstrate the usefulness of our approach on some examples of probabilistic program loops.
\end{abstract}

\section{Introduction}

Inductive loop invariants are commonly used in program verification to prove properties of loops in (nondeterministic) programs. Abstract interpretation provides a powerful framework to synthesize inductive invariants automatically from the given program text [7]. In this paper, we provide a static analysis framework for probabilistic loops that can call random number generators to sample from pre-specified distributions such as Bernoulli, Uniform Random and Normal (Gaussian). Probabilistic programs arise in a variety of domains ranging from biological systems [18] to randomized algorithms [23. In this paper, we present an abstract interpretation framework for deriving expectation invariants of probabilistic loops. Expectation invariants are expressions whose expectations at any given iteration of the loop exist, and are always non-negative.

Proving expectation invariants often requires approximating the distribution of states after $n$ steps of loop execution (see [2, 20, 22, 9, 17] for techniques that approximate distributions in a sound manner). However, even simple programs, such as the program shown in Figure 1, can exhibit complex distributions of reachable states after just a few steps of loop execution (see Figure 2). Extrapolating from a few to arbitrarily many loop iterations requires the notion of "inductive invariants" for probabilistic programs. In this paper, we build upon the standard notion of quantitative invariants originally considered by McIver and Morgan [19]. First we extend quantitative invariants from single expressions to a set of expressions that are mutually invariant: multiple expressions whose expectations are nonnegative simultaneously. Next, we characterize invariants as a fixed point, making them amenable to automatic approximation using abstract interpretation. We demonstrate polyhedral analysis over numerical probabilistic programs that manipulate real- and integer-valued state variables.

\footnotetext{
*first.lastname@colorado.edu

${ }^{\dagger}$ first.lastname@colorado.edu
} 
Our approach first defines the notion of inductive invariants using the pre-expectation operator, along the lines of McIver and Morgan [19]. We lift the pre-expectation operator to a cone of expressions, and subsequently construct a monotone operator over finitely generated cones. Any pre-fixed point of this monotone operator is shown to correspond to expectation invariants. We then use the descending abstract Kleene iteration starting from the cone $T$ of all affine (or fixed degree polynomial expressions) to iteratively apply the monotone operator to this cone and obtain a pre-fixed point. A (dual) widening operator is used to accelerate this process.

We apply our technique to some small but complex examples of probabilistic programs and demonstrate the power of our approach to synthesize expectation invariants that are otherwise hard to realize manually. We also compare our approach with the tool PRINSYS that synthesizes quantitative invariants using a constraint-based approach by solving constraints on the unknown coefficients of a template invariant form [13, 11].

Related Work. The broader area of probabilistic program analysis has seen much progress over the recent past. Our previous work combining symbolic execution of probabilistic programs with volume computation, provides an extensive review of approaches in this area [24]. Therefore, we restrict ourselves to very closely related works.

McIver and Morgan were among the first to consider deductive approaches for probabilistic programs using the concept of quantitative invariants [19]. Their work focuses on programs where the stochastic inputs are restricted to discrete distributions over a finite set of support. We naturally lift this restriction to consider a richer class of distributions in this paper including Gaussian, Poisson, Uniform or Exponential random variables. Our setup can use any distributions whose expectations (and some higher moments) exist, and are available. Furthermore, our technique synthesizes invariants that are polynomial expressions involving the program variables. In particular, indicator functions over program assertions are not considered in this paper [13, 19]. Indicator functions complicate the computation of the preexpectation when a richer class of distributions are allowed. Finally, McIver \& Morgan treat demonic non-deterministic as well as stochastic inputs. Our approach, currently, does not support (demonic) non-determinism; but is potentially extensible when demonic non-determinism is present. Our previous work [3] first considered the relationship between quantitative invariants and the well-known concept of martingales and super-martingales from probability theory [26]. In particular, it demonstrates the use of concentration of measure inequalities to prove probability bounds on assertions at various points in the program [10]. The notion of inductive expectation invariants is a strict generalization of that considered in our previous work. While martingales and super-martingales are analogous to a single inductive linear inequality, we consider the analog of multiple mutually inductive linear invariants. The use of abstract interpretation framework is an additional contribution. The generation of quantitative invariants was first studied by Katoen et al. [13, using a constraint-based approach [6, 25], implemented in the tool PRINSYS [11]. An experimental comparison is provided in Section 5 .

Abstract domains for probabilistic programs were first considered by Monniaux [20], by enriching standard abstract domains with bounds on the measure. Refinements of this idea appear in the work of Mardziel et al [17] and Bouissou et al. [2]. Instead of the explicit representations of distributions found in these works, we characterize sets of distributions by means of bounds on moments of expressions. Alternatively, Monniaux presents a backward abstract interpretation scheme to compute the probability of an observable assertion at the program output, and characterize the output distribution [21]. The backwards approach treats the program as a measurable function, and the backward abstract interpretation follows the natural definition of the output distribution through the inverse mapping [5]. However, the approach seemingly requires a user generated query or a systematic gridding of the output states to define the distribution. Cousot and Monerau [9] present a systematic and general abstract semantics for probabilistic programs that views the abstract probabilistic semantics obtained by separately considering abstractions of the program semantics, the probability (event) space, and a "law abstraction" that is a function mapping abstract states to the distribution over the set of possible abstract next states obtained from a single step of program execution. Their approach conveniently captures existing techniques as 
Section 4 presents an abstract interpretation based iterative approach to compute fixed points under the pre-expectation operator. Section 5 is a summary of the experiments we conducted using our prototype version of the tool and a comparison with the PRINSYS tool.

\section{Preliminaries}

\subsection{Probabilistic Loops}

Let $\mathcal{P}$ be a probabilistic program in an imperative language with random number generators including unifInt (lb, ub), unifReal (lb, ub), and gaussian(mean, var). These constructs draw values from standard distributions with well-defined, finite expected values. Let $X=\left\{x_{1}, \ldots, x_{m}\right\}$ be a set of realvalued program variables and $R=\left\{r_{1}, \ldots, r_{l}\right\}$ be a set of real-valued random variables. Vectors $\mathbf{x}$ and $\mathbf{r}$ denote valuations of all program, respectively random, variables. The random variables have a joint distribution $\mathcal{D}_{R}$. Formally, the distribution is defined over an underlying $\sigma$-algebra $(\Omega, \mathcal{F})$ with an appropriate measure $\mu_{r}$.

A linear inequality over $X$ is an expression of the form $\mathbf{a}^{T} \mathbf{x} \leq b$ for a vector $\mathbf{a} \in \mathbb{R}^{m}, b \in \mathbb{R}$. A linear assertion $\varphi[X]$ involving $X$ is a conjunction of linear inequalities $\varphi: \bigwedge_{i=1}^{n} \mathbf{a}_{i}^{T} \mathbf{x} \leq b_{i}$ and can be succinctly expressed in matrix notation as $\varphi: A \mathbf{x} \leq \mathbf{b}$.

Definition 2.1 (Probabilistic Loops). A probabilistic loop is a tuple $\left\langle\mathcal{T}, \mathcal{D}_{0}, n\right\rangle$, wherein $\mathcal{T}:\left\{\tau_{1}, \ldots, \tau_{k}\right\}$ is a set of probabilistic transitions (from the loop head to itself), $\mathcal{D}_{0}$ is the initial probability distribution and $n$ is a formal loop counter variable.

Each probabilistic transition $\tau_{i}:\left\langle\mathbf{g}_{i}, \mathcal{F}_{i}\right\rangle$ consists of (a) guard assertion $\mathbf{g}_{i}[X]$ over $X$; and (b) update function $\mathcal{F}_{i}(\mathbf{x}, \mathbf{r})$ that yields the next state $\mathbf{x}^{\prime}:=\mathcal{F}_{i}(\mathbf{x}, \mathbf{r})$.

In this paper, we restrict ourselves to piecewise linear (PWL) probabilistic programs, wherein each transition $\tau_{i}$ has linear assertion guards and piecewise linear updates. Further, we also restrict ourselves to studying expectation invariants over simple loops. An extension of these ideas to programs with arbitrary control flow structure including nested loops we reserve as future work.

Definition 2.2 (PWL Transitions). A piecewise linear transition $\tau:\langle\mathbf{g}, \mathcal{F}(\mathbf{x}, \mathbf{r})\rangle$ has the following special structure:

- $\mathrm{g}$ is a linear guard assertion over $X$;

- $\mathcal{F}(\mathbf{x}, \mathbf{r})$ is a (continuous) piecewise linear update function for $X$, where, for ease of presentation, $\mathbf{r}$ is decomposed into a vector of continuous (random) choices $\mathbf{r}_{c}$ and a vector of discrete Bernoulli choices (coin flips) $\mathbf{r}_{b}$. As a result, the update function may be written as

$$
F(\mathbf{x}, \mathbf{r})=\left\{\begin{array}{cc}
\mathrm{f}_{1}: A_{1} \mathbf{x}+B_{1} \mathbf{r}_{c}+d_{1}, & \text { with probability } p_{1}, \\
\vdots & \\
\mathrm{f}_{\mathrm{k}}: A_{k} \mathbf{x}+B_{k} \mathbf{r}_{c}+d_{k}, & \text { with probability } p_{k},
\end{array}\right.
$$

Options $\mathrm{f}_{1}, \ldots, \mathrm{f}_{\mathrm{k}}$, abstract the effect of the Bernoulli choices in $\mathbf{r}_{b}$, and are called forks, while $p_{1}, \ldots, p_{k}$ are fork probabilities satisfying $0<p_{i} \leq 1$, and $\sum_{i=1}^{k} p_{i}=1$; with $A_{1}, \ldots, A_{k} \in \mathbb{R}^{m \times m}$, $B_{1}, \ldots, B_{k} \in \mathbb{R}^{m \times l}$, and $d_{1}, \ldots, d_{k} \in \mathbb{R}$.

No Nondeterminism. For a probabilistic loop $\left\langle\mathcal{T}, \mathcal{D}_{0}, n\right\rangle$, we preclude demonic nondeterminism using two restrictions:

Mutual Exclusion: For all pairs of transitions $\tau_{1}:\left\langle\mathbf{g}_{1}, \mathcal{F}_{1}\right\rangle$ and $\tau_{2}:\left\langle\mathbf{g}_{2}, \mathcal{F}_{2}\right\rangle$ in $\mathcal{T}, \mathbf{g}_{1} \wedge \mathbf{g}_{2} \equiv$ false.

Exhaustiveness: For all transitions $\tau_{i}$, we require that $\bigvee_{\tau_{i} \in \mathcal{T}} \mathbf{g}_{i} \equiv$ true. 
Mutual exclusion and mutual exhaustiveness together guarantee that precisely one transition can be taken at a time step $n$ and the choice is a function of the state $\mathbf{x}$.

Execution Model. A state of the probabilistic loop is a tuple $\langle\mathbf{x}, n\rangle$ that provides values for the program variables $X$ and the loop counter $n$. The state $\left\langle\mathbf{x}_{0}, 0\right\rangle$ is called an initial state if $\mathbf{x}_{0}$ is a sample drawn from the initial distribution $\mathcal{D}_{0}$ and $n=0$.

Definition 2.3 (Sample Path). A sample path (or an execution) of the loop is an infinite sequence $\left(\mathbf{x}_{0}, 0\right) \stackrel{\tau^{(0)}, \mathbf{r}_{0}}{\longrightarrow}\left(\mathbf{x}_{1}, 1\right) \stackrel{\tau^{(1)}, \mathbf{r}_{1}}{\longrightarrow}\left(\mathbf{x}_{2}, 2\right) \rightarrow \cdots \stackrel{\tau^{(n)}, \mathbf{r}_{n}}{\longrightarrow}\left(\mathbf{x}_{n+1}, n+1\right) \rightarrow \cdots$, wherein, (a) $\left(\mathbf{x}_{0}, 0\right)$ is a sample from $\mathcal{D}_{0}$ and (b) for each $i \geq 0,\left(\mathbf{x}_{i+1}, i+1\right)$ is obtained by executing the unique transition $\tau^{(i)}:\left(\mathbf{g}_{i}, \mathcal{F}_{i}\right)$ that is enabled on the state $\left(\mathbf{x}_{i}, i\right)$. This execution involves a sample from the Bernoulli (discrete) random variables to choose a fork of the transition $\tau^{(i)}$ and a choice of the continuous random variables $\mathbf{r}_{c}$ to obtain $\mathbf{x}_{i+1}=\mathcal{F}_{i}\left(\mathbf{x}_{i}, \mathbf{r}_{i}\right)$.

We demonstrate the definitions above on a simple example.

Example 2.1. In Figure 1 (Left, Bottom) we present the stuttering version of a simple probabilistic program with a loop, where the initial values of the program variables reaching the loop head come from the joint distribution $\mathcal{D}_{0}:\langle\mathrm{x}, \mathrm{y}$, count $\rangle \sim U[-5,3] \times U[-3,5] \times\{0\}$. This stuttering modification adds a new program path that preserves the values of program variables once the loop guard $\mathrm{x}+\mathrm{y} \leq 10$ is violated. The program has two transitions $\mathcal{T}:\left\{\tau_{1}, \tau_{2}\right\}$, where $\tau_{1}$ represents the loop body:

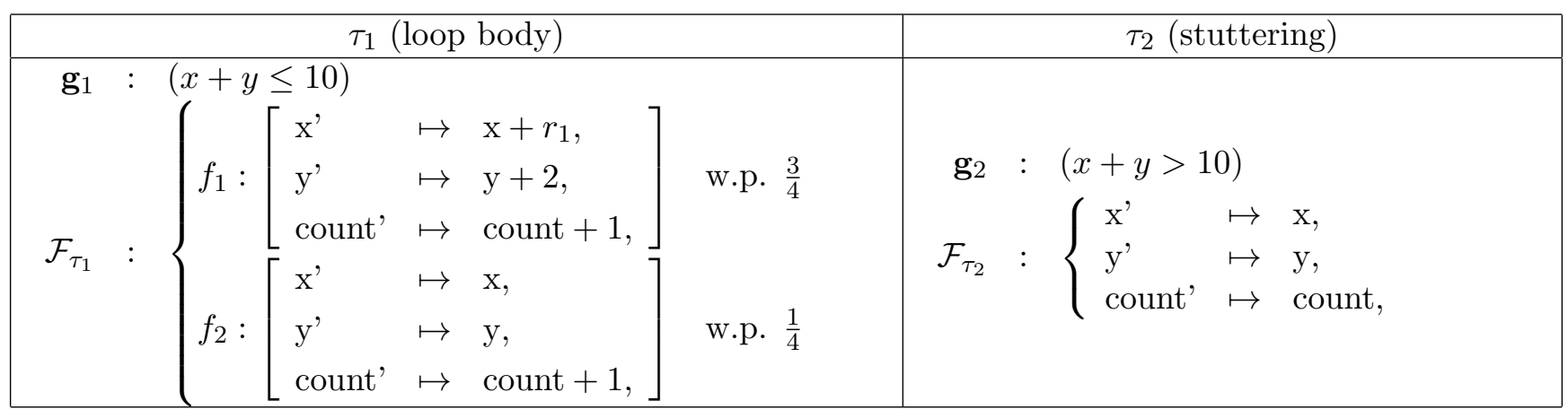

Here $r_{1}$ represents the uniform random variable over [0,2]. Transition $\tau_{2}$ represents the stuttering after $x+y>10$. It is added to satisfy the mutual exclusiveness and exhaustiveness requirements. It has a single fork that preserves the values of $\mathrm{x}, \mathrm{y}$, count. Figure 2 depicts 200 sample paths obtained by simulating the program (for 25 steps) and distributions $\mathcal{D}_{n}$ for $n=0$ and $n=25$ obtained by running the program $10^{6}$ times.

\subsection{Operator Semantics}

Probabilistic program semantics can be thought of as continuous linear operators over the state distributions, starting from the initial distribution $\mathcal{D}_{0}$ :

$$
\mathcal{D}_{0} \stackrel{\llbracket \mathcal{P} \rrbracket}{\longrightarrow} \mathcal{D}_{1} \stackrel{\llbracket \mathcal{P} \rrbracket}{\longrightarrow} \cdots \stackrel{\llbracket \mathcal{P} \rrbracket}{\longrightarrow} \mathcal{D}_{n} \stackrel{\llbracket \mathcal{P} \rrbracket}{\longrightarrow} \cdots
$$

Here, $\llbracket \mathcal{P} \rrbracket$ models the effect of a single loop iteration and $\mathcal{D}_{n}$ is the distribution of the states after $n$ iterations of the loop. This matches the standard probabilistic program semantics [15, 21].

We now describe the construction of the distribution $\mathcal{D}_{n+1}$ for the $(n+1)^{t h}$ loop iteration, given the distribution $\mathcal{D}_{n}$ at the $n^{\text {th }}$ step. The details of this section may be skipped upon a first reading.

Formally, $\mathcal{D}_{n}$ consists of a probability space $\left\langle\Omega_{n}, \mathcal{F}_{n}, \mu_{n}\right\rangle$ over a sample set $\Omega_{n}$ with events in $\mathcal{F}_{n}$ and a probability measure $\mu_{n}$, as well as a Borel measurable function $D_{n}: \Omega_{n} \rightarrow \mathbb{R}^{m}$ mapping samples $\omega \in \Omega_{n}$ 

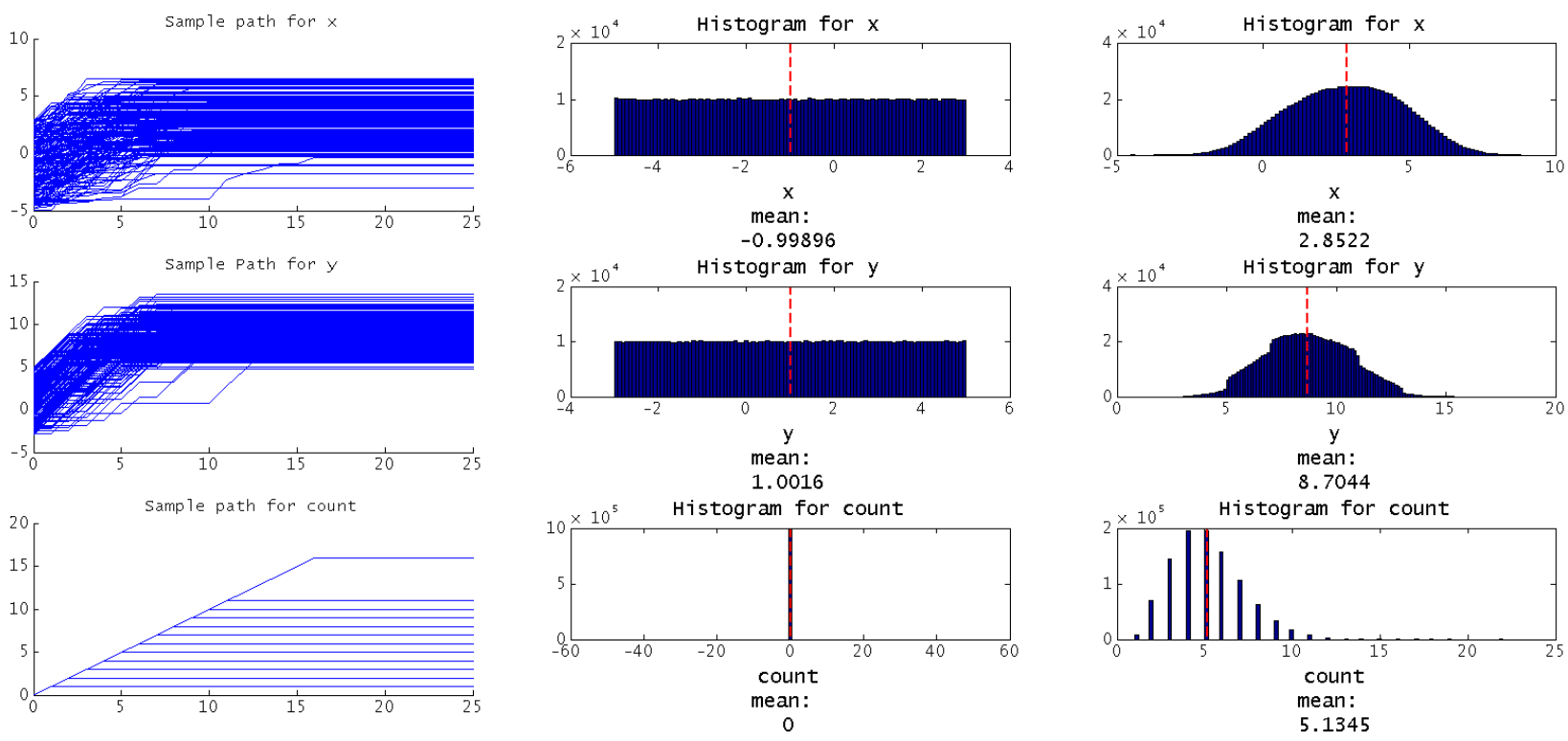

Figure 2: (Left) Some sample paths for the program in Figure1. (Right) Frequency histograms for the distributions $\mathcal{D}_{n}$ for $\mathrm{n}=0,25$.

to values of the state variable $\mathbf{x}$. Likewise, the distribution of random $\mathbf{r}$ is given by $\mathcal{D}_{R}$, defined as the probability space $\left\langle\Omega_{r}, \mathcal{F}_{r}, \mu_{r}\right\rangle$ and a measurable function $D_{r}: \Omega_{r} \rightarrow \mathbb{R}^{k}$ mapping samples $\omega_{r} \in \Omega_{r}$ to random variables $\mathbf{r}$.

The standard product measure $\mathcal{D}_{n, R}: \mathcal{D}_{n} \otimes \mathcal{D}_{R}$ has sample set $\Omega_{n+1}: \Omega_{n} \times \Omega_{r}$ and events $\mathcal{F}_{n, R}$ generated by sets of the form $A \times B$ where $A \in \mathcal{F}_{n}$ and $B \in \mathcal{F}_{r}$ with a measurable function $D_{n, r}$ : $\Omega_{n} \times \Omega_{r} \rightarrow \mathbb{R}^{m} \times \mathbb{R}^{k}$, mapping events $\left(\omega_{n}, \omega_{r}\right)$ to corresponding values of state and random variables $\left(\mathbf{x}: D_{n}\left(\omega_{n}\right), \mathbf{r}: D_{r}\left(\omega_{r}\right)\right)$.

The distribution $\mathcal{D}_{n+1}$ is defined naturally by the product probability space $\mathcal{D}_{n, R}$ with a measurable function $D_{n+1}: \Omega_{n} \times \Omega_{r} \mapsto \mathbb{R}^{m}$ maps a sample $\left(\omega_{n}, \omega_{r}\right)$ from the sample space $\Omega_{n} \times \Omega_{r}$ to the state $\mathbf{x}^{\prime}$ that results from the execution of the probabilistic loop for a single step starting from the state $\mathbf{x}: D_{n}\left(\omega_{n}\right)$ and random values $\mathbf{r}: D_{r}\left(\omega_{r}\right)$.

$$
D_{n+1}\left(\omega_{n}, \omega_{r}\right):\left\{\mathbf{x}^{\prime} \mid \mathbf{x}=D_{n}\left(\omega_{n}\right), \mathbf{r}=D_{r}\left(\omega_{r}\right), \mathbf{x} \stackrel{\mathbf{r}}{\rightarrow} \mathbf{x}^{\prime}\right\} .
$$

The absence of nondeterminism in our setup ensures that $\mathbf{x}^{\prime}$ is uniquely defined given samples $\omega_{n}$, and $\omega_{r}$. To summarize, the next state distribution $\mathcal{D}_{n+1}$ has a probability space defined as the product probability space of $\mathcal{D}_{n} \otimes \mathcal{D}_{r}$ with a measurable function $D_{n+1}$ that maps samples from $\mathcal{D}_{n}$ and $\mathcal{D}_{r}$ to the resulting states obtained by a single step execution of the loop.

Sample Path Semantics: Equivalently, a direct characterization of the distribution $\mathcal{D}_{n}$ is given as the product of the probability spaces

$$
\mathcal{D}_{0} \otimes \underbrace{\mathcal{D}_{R} \otimes \cdots \otimes \mathcal{D}_{R}}_{n \text { times }}
$$

equipped with a measurable function $F_{n}: \Omega_{0} \times \Omega_{r}^{n} \mapsto \mathbb{R}^{m}$ that maps each sample $\hat{\omega}:\left(\omega_{0}, \omega_{r 1}, \ldots, \omega_{r n}\right)$ to the unique resulting state $\mathbf{x}_{n}$ obtained by starting from initial state $\mathbf{x}_{0}: D_{0}\left(\omega_{0}\right)$ and executing the loop $n$ times with a series of random values $\mathbf{r}_{1}: D_{r}\left(\omega_{r 1}\right), \ldots \mathbf{r}_{n}: D_{r}\left(\omega_{r n}\right)$, at each iteration to yield a sample path:

$$
\mathbf{x}_{0}: D_{0}\left(\omega_{0}\right) \stackrel{\mathbf{r}_{1}: D_{r}\left(\omega_{r 1}\right)}{\longrightarrow} \mathbf{x}_{1} \stackrel{\mathbf{r}_{2}: D_{r}\left(\omega_{r 2}\right)}{\longrightarrow} \cdots \stackrel{\mathbf{r}_{n}: D_{r}\left(\omega_{r n}\right)}{\longrightarrow} \mathbf{x}_{n}
$$


The absence of nondeterminism ensures that the sample path is unique once $\mathbf{x}_{0}, \mathbf{r}_{1}, \ldots, \mathbf{r}_{n}$ are known.

Existence of Moments We assume that the distributions $\mathcal{D}_{0}$ and $\mathcal{D}_{R}$ are independent, and all moments exist (and are finite). Therefore, for any polynomial $p\left(\mathbf{x}_{0}, \mathbf{r}_{1}, \ldots, \mathbf{r}_{m}\right)$ wherein $\mathbf{x}_{0} \sim \mathcal{D}_{0}$ and $\mathbf{r}_{i} \sim \mathcal{D}_{R}$, with variables $\mathbf{r}_{i}, \mathbf{r}_{j}$ pairwise independent for $i \neq j, \mathbb{E}_{\mathcal{D}_{0} \otimes \mathcal{D}_{R} \cdots \otimes \mathcal{D}_{R}}(p)$ exists (and is finite).

Let $\mathcal{P}$ be a piecewise linear probabilistic program and $\mathrm{e}\left(\mathbf{x}_{n}\right)$ be a polynomial expression over the variables $\mathbf{x}_{n}$ obtained after $n$ steps of loop execution. Under the assumptions above on only $\mathcal{D}_{0}$ and $\mathcal{D}_{r}$, we conclude the following key result:

Lemma 2.1. For any polynomial expression $\mathrm{e}(\mathbf{x})$ over the program variables, and any $n \in \mathbb{N}, \mathbb{E}_{\mathcal{D}_{n}}(\mathrm{e})$ exists (and is finite).

\subsection{Pre-Expectations}

We now define the useful concept of pre-expectation of an expression e over the program variables across a transition $\tau$ following earlier work by McIver and Morgan [19]. Let $\tau:\langle\mathbf{g}, \mathcal{F}\rangle$ be a transition and e[x] be an expression involving the state variables $\mathbf{x}$ of the program.

Given any state $\mathbf{x}$ of the loop and an expression e involving the state variables, we seek to know the expected value of the expression $\mathrm{e}\left(\mathbf{x}^{\prime}\right)$ evaluated over all possible next states $\mathbf{x}^{\prime}$ that can be reached in one loop iteration starting from $\mathbf{x}$. The resulting function that maps $\mathbf{x}$ to the expectation of the e in the next step is called the pre-expectation of e, following the terminology established earlier by McIver and Morgan [19]. We first defined the pre-expectation w.r.t a given transition $\tau$ and then use this to define the pre-expectation w.r.t to all transitions in the loop.

The pre-expectation operator pre $\mathbb{E}_{\tau}$ of an expression e involving the next-state program variables $X^{\prime}$, w.r.t to a transition $\tau$ is an expression transformer that computes the expectation of the expression $\mathbf{e}\left(\mathbf{x}^{\prime}\right)$ in the next step in terms of current state variables of the program $\mathbf{x}$. Formally,

$$
\operatorname{pre} \mathbb{E}_{\tau}\left(\mathrm{e}\left[\mathbf{x}^{\prime}\right]\right): \mathbb{E}_{R}\left(\mathrm{e}\left[\mathbf{x}^{\prime} \mapsto \mathcal{F}(\mathbf{x}, \mathbf{r})\right] \mid \mathbf{x}\right)
$$

The expectation $\mathbb{E}_{R}$ is taken over the distribution of $\mathbf{r}$ along transition $\tau$.

Notation: We define the pre-expectation as a map that transforms expressions over the next-state variables $X^{\prime}$ into current state variable expression $X$. Therefore, wherever applicable, pre $\mathbb{E}\left(\mathrm{e}\left(\mathrm{x}^{\prime}\right)\right)$ results in an expression over the original state variables $\mathbf{x}$. For convenience, let $\mathrm{e}^{\prime}$ denote the expression $\mathrm{e}\left[\mathbf{x} \mapsto \mathbf{x}^{\prime}\right]$ with each current state variable $x_{j}$ substituted by its next-state variable $x_{j}^{\prime}$.

Consider a PWL transition $\tau$ with $k>0$ forks, $\mathrm{f}_{1}, \ldots, \mathrm{f}_{\mathrm{k}}$, each of the form $\mathrm{f}_{\mathrm{j}}: A_{j} \mathbf{x}+B_{j} \mathbf{r}+d_{j}$ with fork probability $p_{j}$. The pre-expectation operator is defined as

$$
\operatorname{pre} \mathbb{E}_{\tau}\left(\mathrm{e}^{\prime}\right)=\sum_{j=1}^{k} p_{j} \mathbb{E}_{R}\left(\operatorname{PRE}\left(\mathrm{e}^{\prime}, f_{j}\right) \mid \mathbf{x}\right)
$$

where $\operatorname{PRE}\left(\mathrm{e}^{\prime}, f_{j}\right)$ is the substitution of post variables $\mathbf{x}^{\prime}$ for their update values $f_{j}(\mathbf{x}, \mathbf{r})$ in expression e. The expectation $\mathbb{E}_{R}(g)$ denotes the expectation of $g$ over the joint distribution $\mathcal{R}$ of the random variables.

Example 2.2. We illustrate the notion of a pre-expectation of a program expression by considering the expression $3+2 \mathrm{x}-\mathrm{y}$ across transition $\tau_{1}$ in the Figure 1 .

$$
\operatorname{pre}_{\tau_{1}}\left(3+2 \mathrm{x}^{\prime}-\mathrm{y}^{\prime}\right):\left(\begin{array}{ll}
\frac{3}{4}\left[3+2 \mathbb{E}_{r_{1}}\left(x+r_{1}\right)-(y+2)\right]+ & / / \text { from fork } f_{1} \\
\frac{1}{4}[3+2 x-y] & / / \text { from fork } f_{2}
\end{array}\right) .
$$

Simplifying, we obtain pre $\mathbb{E}_{\tau_{1}}\left(3+2 \mathrm{x}^{\prime}-\mathrm{y}^{\prime}\right)=3+2 x-y+\frac{3}{2} \mathbb{E}_{r_{1}}\left(r_{1}\right)-\frac{3}{2}$. Noting that $\mathbb{E}_{r_{1}}\left(r_{1}\right)=1$, we obtain pre $\mathbb{E}_{\tau_{1}}\left(3+2 \mathrm{x}^{\prime}-\mathrm{y}^{\prime}\right)=3+2 \mathrm{x}-\mathrm{y}$. 
Likewise, we define pre $\mathbb{E}\left(\mathrm{e}^{\prime}\right)$ (without a transition as a subscript) as

$$
\mathbb{1}_{g_{\tau_{1}}} \times \operatorname{pre}_{\tau_{1}}\left(\mathbf{e}^{\prime}\right)+\cdots+\mathbb{1}_{g_{\tau_{k}}} \times \operatorname{pre}_{\tau_{k}}\left(\mathbf{e}^{\prime}\right),
$$

wherein $\mathbb{1}_{g}(\mathbf{x})$ is the indicator function:

$$
\mathbb{1}_{g}(\mathbf{x})= \begin{cases}1 & \text { if } \mathbf{x} \models g(\mathbf{x}) \\ 0 & \text { otherwise }\end{cases}
$$

We now state a key result involving pre-expectations. Consider a prefix $\sigma$ of a sample execution $\left(\mathbf{x}_{0}, 0\right) \rightarrow\left(\mathbf{x}_{1}, 1\right) \rightarrow \cdots \rightarrow\left(\mathbf{x}_{n}, n\right)$. Given that the current state is $\left(\mathbf{x}_{n}, n\right)$, we wish to find out the expectation of an expression e over the distribution of all possible next states $\left(\mathbf{x}_{n+1}, n+1\right)$. Let $\hat{\mathrm{e}}$ : $\operatorname{pre} \mathbb{E}\left(\mathrm{e}^{\prime}\right)$.

Lemma 2.2. The expected value of e over the post-state distribution starting from state $\left(\mathbf{x}_{n}, n\right)$ is the value of the pre-expectation ê evaluated over the current state $\mathbf{x}_{n}$ :

$$
\mathbb{E}\left(\mathrm{e}\left(\mathbf{x}_{n+1}\right) \mid \mathbf{x}_{n}, n\right)=\hat{\mathrm{e}}\left(\mathbf{x}_{n}\right)=\sum_{\tau_{i} \in \mathcal{T}} \mathbb{1}_{g_{\tau_{i}}}\left(\mathbf{x}_{n}\right) \times \operatorname{pre} \mathbb{E}_{\tau_{i}}\left(\mathrm{e}^{\prime}\right) .
$$

Proof. This follows directly from the definition of the pre-expectation operator.

Finally, we extend Lemma 2.2 to the full distribution $\mathcal{D}_{n}$ from which $\mathbf{x}_{n}$ is drawn.

Lemma 2.3. Let e be an affine program expression. Then

$$
\mathbb{E}_{\mathcal{D}_{n+1}}(\mathrm{e})=\mathbb{E}_{\mathcal{D}_{n}}\left(\operatorname{pre} \mathbb{E}\left(\mathrm{e}^{\prime}\right)\right)=\mathbb{E}_{\mathcal{D}_{n}}\left[\sum_{\tau_{i} \in \mathcal{T}} \mathbb{1}_{g_{\tau_{i}}} \times \operatorname{pre} \mathbb{E}_{\tau_{i}}\left(\mathrm{e}^{\prime}\right)\right]
$$

\section{$3 \quad$ Expectation Invariants}

Expectation invariants are invariant inequalities on the expected value of program expressions. Therefore, one could view the set of possible state distributions $\mathcal{D}_{i}$ at step $i$ as the concrete domain over which our analysis operates to produce the abstract facts in the form of expectation invariants over these distributions. We formalize the notion of expectation invariants and derive a fixed point characterization of expectation invariants in the next section.

\subsection{Definitions and Examples}

Let $\mathcal{P}:\left\langle\mathcal{T}, \mathcal{D}_{0}, n\right\rangle$ be a probabilistic loop and let $\left\langle\mathbf{x}_{0}, 0\right\rangle$ be the initial state of the system. From Section 2 we know that $\mathbf{x}_{0}$ is drawn from an initial distribution $\mathcal{D}_{0}$ and that any $n$-step sample execution of $\mathcal{P}$ defines a sample trajectory through the distributions of reachable states $\mathcal{D}_{0}, \ldots, \mathcal{D}_{n}$ at step $i$ for any $0 \leq i \leq n$. We then define the expectation of a program expression e at time step $n$ as $\mathbb{E}(\mathrm{e} \mid n)=\mathbb{E}_{\mathcal{D}_{n}}(\mathrm{e})$.

Notation: We denote the expectation of an expression e over the program variables at the $j^{\text {th }}$ step as $\mathbb{E}(\mathrm{e} \mid n=j)$ or equivalently $\mathbb{E}_{\mathcal{D}_{j}}(\mathrm{e})$. Unless otherwise mentioned, e will denote an affine (or linear) expression over the program variables.

Definition 3.1 (Expectation Invariants). An e over the program variables $X$ is called an expectation invariant (EI) iff for all $n \geq 0, \mathbb{E}(\mathrm{e} \mid n) \geq 0$.

Thus, expectation invariants are program expressions whose expectations over the initial distribution are non-negative, and under any number $n \geq 0$ of iterations of the probabilistic loop remain non-negative.

Example 3.1. Consider the program from Example 1, and the expression $y-x$. Initially, $\mathbb{E}(\mathrm{y}-\mathrm{x} \mid 0)=$ $\mathbb{E}_{\mathcal{D}_{0}}(\mathrm{y}-\mathrm{x})=1-(-1)=2 \geq 0$. We can show that $\mathbb{E}(\mathrm{y}-\mathrm{x} \mid i)=\mathbb{E}(\mathrm{y} \mid i)-\mathbb{E}(\mathrm{x} \mid i) \geq 0$ at any step $i$. Therefore, $\mathrm{y}-\mathrm{x}$ is an expectation invariant. 


\subsection{Martingales and Expectation Invariants}

Expectation invariants as given by Definition 3.1 are closely related to the concept of (super-, sub-) martingales, studied in our earlier work [3].

Definition 3.2 (Martingales). Let $s:\left\langle\mathbf{x}_{n}, n\right\rangle$ be the state of a probabilistic loop $\mathcal{P}$. Let e be a program expression over the program variables $X$. Expression e is called:

- a supermartingale if $\forall n, \mathbf{x}, \operatorname{pre} \mathbb{E}\left(\mathrm{e}^{\prime}\right) \leq \mathrm{e}$,

- a submartingale if $\forall n, \mathbf{x}$, pre $\mathbb{E}\left(\mathrm{e}^{\prime}\right) \geq \mathrm{e}$,

- a martingale if e is both a supermartingale and a submartingale.

In fact, martingales naturally yield expectation invariants.

Lemma 3.1. For every supermartingale expression e the expression $e_{0}-\mathrm{e}$ is an expectation invariant, wherein $e_{0}=\mathbb{E}(\mathrm{e} \mid n=0)$.

Proof. First, we observe that $\forall \mathbf{x}, \operatorname{pre} \mathbb{E}\left(\mathrm{e}^{\prime}\right) \leq$ e. Therefore, assuming that the expectations on both sides exist, we have for all $n \geq 0, \mathbb{E}_{\mathcal{D}_{n}}\left(\operatorname{pre} \mathbb{E}\left(\mathrm{e}^{\prime}\right)\right) \leq \mathbb{E}_{\mathcal{D}_{n}}(\mathrm{e})$.

We now prove that $\mathbb{E}\left(e_{0}-\mathrm{e} \mid n\right) \geq 0$ for all $n \in \mathbb{N}$ by induction. Clearly, for $n=0$, the statement holds. Furthermore, assume that $\mathbb{E}\left(e_{0}-\mathrm{e} \mid n=j\right)$ holds, we obtain

$$
\begin{aligned}
\mathbb{E}\left(e_{0}-\mathrm{e} \mid n=j+1\right) & =\mathbb{E}\left(e_{0}-\operatorname{pre} \mathbb{E}\left(\mathrm{e}^{\prime}\right) \mid n=j\right) \\
& =e_{0}-\mathbb{E}\left(\operatorname{pre} \mathbb{E}\left(\mathrm{e}^{\prime}\right) \mid n=j\right) \\
& \geq e_{0}-\mathbb{E}(\mathrm{e} \mid n=j) \geq 0 .
\end{aligned}
$$

However, expectation invariants can arise without martingales, as shown by the following simple example that repeatedly swaps two variables $\mathrm{x}, \mathrm{y}$ :

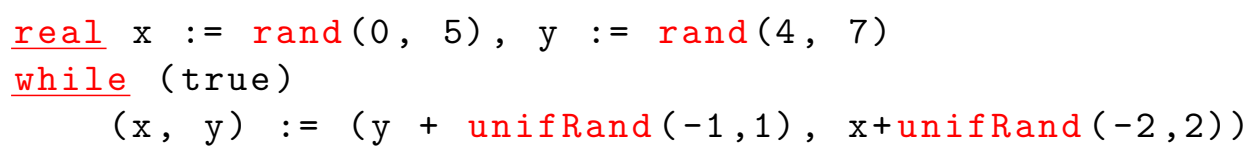

Notice that expressions $\mathrm{x}$ and $\mathrm{y}$ are expectation invariant. However, they are not martingales. In fact, to prove that $\mathbb{E}(\mathrm{x}) \geq 0$, at any step, we require that $\mathbb{E}(\mathrm{y}) \geq 0$ at the previous step.

Therefore, the notion of expectation invariants subsumes that of martingales as defined here. Drawing analogies to the familiar case of Floyd-Hoare invariants, martingales correspond to assertions which are invariant by themselves, whereas expectation invariants are analogous to the general case of mutually inductive invariants [16].

\subsubsection{Proving Expectation Invariance}

We now focus on the question of proving that a given expression e over the program variables is an expectation invariant. This requires constructing (approximations) to the distribution $\mathcal{D}_{n}$ for each $n$, or alternatively, an argument based on mathematical induction. We first observe an important property of each $\mathcal{D}_{n}$.

Definition 3.3 (Admissible Distribution). We say that a distribution $\mathcal{D}$ over the state-space $\mathcal{X}$ is admissible if all moments exist ${ }^{1}$ In other words, for any polynomial $p(\mathbf{x})$ over the program variables, $\mathbb{E}_{\mathcal{D}}(p(\mathbf{x}))$ exists, and is finite.

\footnotetext{
${ }^{1}$ While the existence of only the first moment suffices, our experiments demonstrate that our current synthesis approach can be extended to polynomial expectation invariants.
} 
Let us assume that any program $\mathcal{P}$ which we attempt to analyze is such that

1. $\mathcal{D}_{0}$, the initial state distribution, is admissible;

2. For each transition $\tau$, the distribution of the random variables $\mathcal{D}_{R}$ is admissible.

Under these assumptions, we invoke Lemma 2.1 to conclude that $\mathcal{D}_{n}$ is admissible for each $n \geq 0$. However, rather than construct $\mathcal{D}_{n}$ explicitly for each $n$ (which can be impractical), we formulate the principle of inductive expectation invariants. Consider expressions $E=\left\{\mathrm{e}_{1}, \ldots, \mathrm{e}_{m}\right\}$ wherein each $\mathrm{e}_{i}$ is a linear (or polynomial) expression involving the program variables.

Definition 3.4 (Inductive Expectation Invariants). The set $E$ of expressions forms an inductive expectation invariant of the program $\mathcal{P}$ iff for each $\mathrm{e}_{j}, j \in[1, m]$,

1. $\mathbb{E}_{\mathcal{D}_{0}}\left(\mathrm{e}_{j}\right) \geq 0$, i.e., the expectation at the initial step is non-negative.

2. For every admissible distribution $\mathcal{D}$ over the state-space $\mathcal{X}$,

$$
\left(\mathbb{E}_{\mathcal{D}}\left(\mathrm{e}_{1}\right) \geq 0 \wedge \cdots \wedge \mathbb{E}_{\mathcal{D}}\left(\mathrm{e}_{m}\right) \geq 0\right) \models \mathbb{E}_{\mathcal{D}}\left(\operatorname{pre} \mathbb{E}_{\left.\left(\mathrm{e}_{j}^{\prime}\right)\right) \geq 0}\right.
$$

The inductive expectation invariant principle stated above follows the standard Floyd-Hoare approach of "abstracting away" the distribution at the $n^{\text {th }}$ step by the inductive invariant itself, and using these to show that the invariant continues to hold for one more step. Furthermore, it abstracts away from a specific $\mathcal{D}_{n}$ to any admissible distribution $\mathcal{D}$.

Theorem 3.1. Let $E:\left\{\mathrm{e}_{1}, \ldots, \mathrm{e}_{m}\right\}$ be inductive expectation invariants (Definition 3.4), it follows that each $\mathrm{e}_{j} \in E$ is an expectation invariant of the program:

$$
\forall n \in \mathbb{N}, \mathbb{E}\left(\mathrm{e}_{j} \mid n\right) \geq 0
$$

Proof. The proof uses the important fact that each distribution $\mathcal{D}_{n}$ is admissible. We prove by simultaneous induction that

$$
\bigwedge_{j=1}^{m} \mathbb{E}\left(\mathrm{e}_{j} \mid n\right) \geq 0 .
$$

Base-Case: The base case for $n=0$ follows from item 1 of Definition 3.4 .

Induction Step: Let us assume that the required statement holds for $n$ and attempt to show for $n+1$. Using Eq. (1), and the admissibility of $\mathcal{D}_{n}$, we note that for each $j \in[1, m]$,

$$
\left(\mathbb{E}_{\mathcal{D}_{n}}\left(\mathrm{e}_{1}\right) \geq 0 \wedge \cdots \wedge \mathbb{E}_{\mathcal{D}_{n}}\left(\mathrm{e}_{m}\right) \geq 0\right) \models \mathbb{E}_{\mathcal{D}_{n}}\left(\operatorname{pre} \mathbb{E}\left(\mathrm{e}_{j}^{\prime}\right)\right) \geq 0
$$

Therefore, we conclude that $\mathbb{E}_{\mathcal{D}_{n}}\left(\right.$ pre $\left.\mathbb{E}\left(\mathrm{e}_{j}^{\prime}\right)\right) \geq 0$. Since, $\mathbb{E}_{\mathcal{D}_{n+1}}\left(\mathrm{e}_{j}\right)=\mathbb{E}_{\mathcal{D}_{n}}\left(\operatorname{pre} \mathbb{E}\left(\mathrm{e}_{j}^{\prime}\right)\right)$, we have $\mathbb{E}_{\mathcal{D}_{n+1}}\left(\mathrm{e}_{j}\right)=$ $\mathbb{E}_{\mathcal{D}_{n}}\left(\right.$ pre $\left.\mathbb{E}\left(\mathrm{e}_{j}^{\prime}\right)\right) \geq 0$ for each $j$. Thus, the induction step is proven.

However, Definition 3.4 is quite unwieldy, in practice, since the quantification over all possible admissible distributions $\mathcal{D}$ over the state space $\mathcal{X}$ is a higher order quantifier (over probability spaces and measurable functions). Rather than reason with this quantifier, we will use the following facts about expectations to formulate a new principle:

Theorem 3.2 (Facts About Expectations over Admissible Distributions). The following hold over all possible admissible distributions $\mathcal{D}$ over a $\sigma$-algebra $\mathcal{X}$, linear assertion $\varphi$, and linear (or polynomial expressions) e, $\mathrm{e}_{1}, \ldots, \mathrm{e}_{k}$ :

1. Linearity of expectation: $\mathbb{E}_{\mathcal{D}}\left(\lambda_{1} \mathrm{e}_{1}+\ldots+\lambda_{k} \mathrm{e}_{k}\right)=\lambda_{1} \mathbb{E}_{\mathcal{D}}\left(\mathrm{e}_{1}\right)+\cdots+\lambda_{k} \mathbb{E}_{\mathcal{D}}\left(\mathrm{e}_{k}\right)$, for $\lambda_{i} \in \mathbb{R}$.

2. If $\varphi=\mathrm{e} \geq 0$ then $\mathbb{E}_{\mathcal{D}}\left(\mathbb{1}_{\varphi} \times \mathrm{e}\right) \geq 0$, provided $\llbracket \varphi \rrbracket$ is measurable. Specifically, $\mathbb{E}_{\mathcal{D}}\left(\mathbb{1}_{\mathrm{e} \geq 0} \times \mathrm{e}\right) \geq 0$. 
3. $\mathbb{E}_{\mathcal{D}}\left(\mathbb{1}_{\varphi} \mathrm{e}+\mathbb{1}_{\neg \varphi} \mathrm{e}\right)=\mathbb{E}_{\mathcal{D}}(\mathrm{e})$, provided $\llbracket \varphi \rrbracket$ is measurable.

Using these facts as "axioms", we attempt to reformulate the key step 2 of Definition 3.4 as a simple quantified statement in (first-order) linear arithmetic. Consider, once again, the key statement of the principle (1). The central idea of our approach is to express the pre-expectation pre $\mathbb{E}\left(\mathrm{e}_{j}\right)$ for each $\mathrm{e}_{j} \in E$ as

$$
\operatorname{pre} \mathbb{E}\left(\mathrm{e}_{j}^{\prime}\right)=\sum_{i=1}^{m} \lambda_{j, i} \mathrm{e}_{i}+\sum_{p} \mu_{j, p}\left(\mathbb{1}_{\varphi_{p}} \times g_{p}\right),
$$

wherein $\lambda_{j, i} \geq 0$ and $\mu_{j, p} \geq 0$ are real-valued multipliers, $g_{p}$ are linear expressions over the program variables and $\varphi_{p}$ are assertions such that $\varphi_{p} \models g_{p} \geq 0$. The origin of the expressions $g_{p}$ and assertions $\varphi_{p}$ will be made clear, shortly. Let us fix a finite set of expressions $E=\left\{\mathrm{e}_{1}, \ldots, \mathrm{e}_{m}\right\}$.

Lemma 3.2. Suppose for all $\mathrm{e}_{i} \in E$, the principle (2) holds:

$$
\operatorname{pre} \mathbb{E}\left(\mathrm{e}_{j}^{\prime}\right)=\sum_{i=1}^{m} \lambda_{j, i} \mathrm{e}_{i}+\sum_{p} \mu_{j, p}\left(\mathbb{1}_{\varphi_{p}} \times g_{p}\right),
$$

for some $\lambda_{j, i} \geq 0, \mu_{j, p} \geq 0$ and $\varphi_{p} \models g_{p} \geq 0$, then $E$ satisfies the original induction principle (1):

$$
\text { For all admissible } \left.\mathcal{D},\left(\mathbb{E}_{\mathcal{D}}\left(\mathrm{e}_{1}\right) \geq 0 \wedge \cdots \wedge \mathbb{E}_{\mathcal{D}}\left(\mathrm{e}_{m}\right) \geq 0\right) \models \mathbb{E}_{\mathcal{D}}\left(\operatorname{pre} \mathbb{E}_{\left(\mathrm{e}_{j}^{\prime}\right.}\right)\right) \geq 0 \text {. }
$$

Proof. Let $E$ be such that for each $\mathrm{e}_{i} \in E$, we satisfy (2) as below:

$$
\operatorname{pre} \mathbb{E}\left(\mathrm{e}_{j}^{\prime}\right)=\sum_{i=1}^{m} \lambda_{j, i} \mathrm{e}_{i}+\sum_{p} \mu_{j, p}\left(\mathbb{1}_{\varphi_{p}} \times g_{p}\right),
$$

for some $\lambda_{j, i} \geq 0, \mu_{j, p} \geq 0$ and $\varphi_{p}=g_{p} \geq 0$.

Let $\mathcal{D}$ be any admissible distribution such that $\bigwedge_{j=1}^{m} \mathbb{E}_{\mathcal{D}}\left(\mathrm{e}_{j}\right) \geq 0$. Using, linearity of expectation, we note that

$$
\mathbb{E}_{\mathcal{D}}\left(\sum_{i=1}^{m} \lambda_{j, i} \mathrm{e}_{i}\right)=\sum_{i=1}^{m} \lambda_{j, i} \underbrace{\mathbb{E}_{\mathcal{D}}\left(\mathrm{e}_{i}\right)}_{\geq 0} \geq 0 .
$$

Similarly, applying Theorem 3.2 , we note that $\mathbb{E}_{\mathcal{D}}\left(\mathbb{1}_{\varphi_{p}} \times g_{p}\right) \geq 0$.

$$
\mathbb{E}_{\mathcal{D}}\left(\sum_{p} \mu_{j, p}\left(\mathbb{1}_{\varphi_{p}} \times g_{p}\right)\right)=\sum_{p} \mu_{j, p} \underbrace{\mathbb{E}_{\mathcal{D}}\left(\mathbb{1}_{\varphi_{p}} \times g_{p}\right)}_{\geq 0} \geq 0
$$

Combining, (3) and (4), we note that

$$
\begin{aligned}
\mathbb{E}_{\mathcal{D}}\left(\operatorname{pre} \mathbb{E}\left(\mathrm{e}_{j}^{\prime}\right)\right) & =\mathbb{E}_{\mathcal{D}}\left(\sum_{i=1}^{m} \lambda_{j, i} \mathrm{e}_{i}+\sum_{p} \mu_{j, p}\left(\mathbb{1}_{\varphi_{p}} \times g_{p}\right)\right) & \text { From Stmt. of Thm. } \\
& =\mathbb{E}_{\mathcal{D}}\left(\sum_{i=1}^{m} \lambda_{j, i} \mathrm{e}_{i}\right)+\mathbb{E}_{\mathcal{D}}\left(\sum_{p} \mu_{j, p}\left(\mathbb{1}_{\varphi_{p}} \times g_{p}\right)\right) & \\
& \geq 0 & \text { Applying (3) and (4) }
\end{aligned}
$$




\subsection{Conic Inductive Expectation Invariants}

We now formalize this intuitive notion of inductive invariants using the concept of conic inductive expectation invariants. Let $\mathcal{P}$ be a program with transitions $\mathcal{T}$. Let $\mathbf{g}_{i}$ be a linear assertion representing the guard of the transition $\tau_{i}$. We express $\mathbf{g}_{i}$ as $\bigwedge_{j=1}^{n_{i}} g_{i, j} \geq 0$, wherein $g_{i, j}$ are affine program expressions. Let $\mathbf{g}_{\mathbf{i}}:\left(g_{i, 1} \ldots g_{i, n_{i}}\right)^{T}$ be a vector representing $\mathbf{g}_{i}$. Likewise, let $E=\left\{\mathrm{e}_{1}, \ldots, \mathrm{e}_{m}\right\}$ be a finite set of expressions, we denote the vector of expressions as $\mathbf{e}:\left(\mathrm{e}_{1}, \ldots, \mathrm{e}_{m}\right)^{T}$.

Definition 3.5 (Conic Inductive Expectation Invariants). The finite set $E$ is a conic inductive invariant of the program $\mathcal{P}$ iff for each $\mathrm{e}_{j} \in E$,

1. Initial Condition: $\mathbb{E}_{\mathcal{D}_{0}}\left(\mathrm{e}_{j}\right) \geq 0$ over the initial distribution $\mathcal{D}_{0}$;

2. Induction Step: There exists a vector of multipliers $\lambda_{j} \geq 0$, such that for each transition $\tau_{l}:\left(\mathbf{g}_{l}, \mathcal{F}_{l}\right)$, pre $\mathbb{E}_{\tau_{l}}\left(\mathrm{e}_{j}\right)$ can be expressed as a conic combination of expressions in $E$ and the expressions in $\mathbf{g}_{l}$ :

$$
\underline{\text { For each } \mathrm{e}_{j}}\left(\exists \lambda_{j} \geq 0\right)\left(\forall \tau_{l} \in \mathcal{T}\right)\left(\exists \mu_{l} \geq 0\right) \operatorname{pre}_{\tau_{l}}\left(\mathrm{e}_{j}\right)=\lambda_{j}^{T} \mathbf{e}+\mu_{l}^{T} \mathbf{g}_{l} .
$$

In particular, we note that the order of quantification in Equation (5) is quite important. We note for a given expression $\mathrm{e}_{j}$ the multipliers $\lambda_{j}$ must stay the same across all the transitions $\tau_{l} \in \mathcal{T}$. This will ensure the applicability of the linearity of expectation.

Example 3.2. The set $E=\left\{e_{1}: \mathrm{y}-2 \mathrm{x}, e_{2}: 2 \mathrm{x}-\mathrm{y}+3, e_{3}: 4 \mathrm{x}-3 \operatorname{count}+4, e_{4}:-2 \mathrm{x}+\mathrm{y}-3, e_{5}\right.$ : $-4 \mathrm{x}+3$ count -4$\}$ is a conic inductive invariant for the program in Example 1.1. Consider $\mathrm{e}_{1}: \mathrm{y}-2 \mathrm{x}$. We have

$$
\operatorname{pre} \mathbb{E}_{\tau_{1}}\left(\mathrm{e}_{1}\right): \mathbb{E}_{r_{1}}\left(\frac{3}{4}\left(\mathrm{y}+2-2 \mathrm{x}-2 r_{1}\right)+\frac{1}{4}(\mathrm{y}-2 \mathrm{x})\right)=\mathrm{y}-2 \mathrm{x} .
$$

Likewise, pre $\mathbb{E}_{\tau_{2}}\left(\mathrm{e}_{1}\right): \mathrm{e}_{1}$, since $\tau_{2}$ is a stuttering transition.

Therefore, setting $\lambda:\left(\begin{array}{lllll}1 & 0 & 0 & 0 & 0\end{array}\right)^{T}$, we obtain pre $\mathbb{E}\left(\mathrm{e}_{1}\right): \lambda^{T} \mathbf{e}+\mathbf{0} \times \mathbb{1}_{x+y \leq 10}$.

Changing the order of quantification in Equation 5 makes the rule unsound. In particular, we will address the need to maintain the multipliers $\lambda_{j}$ the same across all transitions. Consider a variant of the Equation (5), as below:

$$
\underline{\text { For each } \mathrm{e}_{j}}\left(\forall \tau_{l} \in \mathcal{T}\right)\left(\exists \lambda_{\mathbf{j}} \geq \mathbf{0}\right)\left(\exists \mu_{l} \geq 0\right) \operatorname{pre}_{\mathbb{\tau}_{l}}\left(\mathrm{e}_{j}^{\prime}\right)=\lambda_{j}^{T} \mathbf{e}+\mu_{l}^{T} \mathbf{g}_{l} .
$$

Such a rule seems like a natural encoding of the implication:

$$
\bigwedge_{j=1}^{m} \mathrm{e}_{j} \geq 0 \wedge \bigwedge_{k=1}^{q} g_{l, k} \geq 0 \models \operatorname{pre} \mathbb{E}_{\tau_{l}}\left(\mathrm{e}_{j}^{\prime}\right) \geq 0 .
$$

The following example demonstrates the unsoundness of the rule (6).

Example 3.3. Consider the program below:

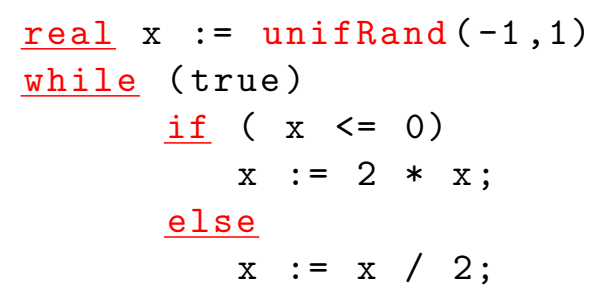

$$
\begin{aligned}
X & :\{\mathrm{x}\} \\
\mathcal{T} & :\left\{\tau_{1}, \tau_{2}\right\} \\
\tau_{1}: & \left\{\begin{array}{l}
\mathbf{g}_{1}: x<0 \\
F_{1}(x): 2 x
\end{array}\right. \\
\tau_{2}: & \left\{\begin{array}{l}
\mathbf{g}_{2}: x \geq 0 \\
F_{2}(x): 0.5 x
\end{array}\right. \\
\mathcal{D}_{0}: & \text { Uniform }[-1,1]
\end{aligned}
$$


First, we observe that $\mathbb{E}_{\mathcal{D}_{0}}(\mathrm{x})=0$. This gives us two IEI candidates $\mathrm{x}$ and $-\mathrm{x}$. Using the rule in Equation 6 we obtain:

- For transition $\tau_{1}$, we have

$$
\operatorname{pre} \mathbb{E}_{\tau_{1}}\left(\mathrm{x}^{\prime}\right)=2 \times(\mathrm{x}), \quad \text { and } \quad \operatorname{pre} \mathbb{E}_{\tau_{1}}\left(-\mathrm{x}^{\prime}\right)=2 \times(-\mathrm{x}) ;
$$

- For transition $\tau_{2}$, we have

$$
\operatorname{pre} \mathbb{E}_{\tau_{2}}\left(\mathrm{x}^{\prime}\right)=0.5 \times(\mathrm{x}), \quad \text { and } \quad \operatorname{pre}_{\tau_{2}}\left(-\mathrm{x}^{\prime}\right)=0.5 \times(-\mathrm{x})
$$

This means that according to rule (6), we can conclude that $\mathbb{E}(\mathrm{x} \mid n) \geq 0$ and $\mathbb{E}(-\mathrm{x} \mid n) \geq 0$, and so $\mathbb{E}(\mathrm{x} \mid n)=0$ for all $n \geq 0$. This is clearly false since any negative initial value of $\mathrm{x}$ only ever execute $\tau_{1}$ and grows unbounded!

The correct version of the rule (5), is able to correctly prove the invariance of $-\mathrm{x}$ and disprove $\mathrm{x}$.

Lemma 3.3. Let $E:\left\{\mathrm{e}_{1}, \ldots, \mathrm{e}_{m}\right\}$ be a conic inductive invariant for a program $\mathcal{P}$ as given by Definition 3.5. It follows that each $\mathrm{e}_{j}$ satisfies Equation (2):

$$
\operatorname{pre} \mathbb{E}\left(\mathrm{e}_{i}^{\prime}\right)=\sum_{j=1}^{n} \lambda_{j, i} \mathrm{e}_{j}+\sum_{p} \mu_{i, p}\left(\mathbb{1}_{\varphi_{p}} \times g_{p}\right),
$$

for $\lambda_{j, i}, \mu_{i, p} \geq 0$ and $\varphi_{p}=g_{p} \geq 0$.

Proof. We note that for each $\mathrm{e}_{i}$, the expression:

$$
\operatorname{pre} \mathbb{E}\left(\mathrm{e}_{i}^{\prime}\right): \sum_{\tau \in \mathcal{T}} \mathbb{1}_{g_{\tau}} \times \operatorname{pre} \mathbb{E}_{\tau}\left(\mathrm{e}_{i}^{\prime}\right)
$$

From (2), there exists $\lambda$ such that for each transition $\tau$,

$$
\operatorname{pre} \mathbb{E}_{\tau}\left(\mathrm{e}_{i}^{\prime}\right)=\lambda^{T} \mathbf{e}+\mu^{T} \mathbf{g}_{\tau} .
$$

Here the guard assertion for $\tau$ is given by $\mathbf{g}_{\tau} \geq 0$. Substituting this into Equation (7) yields,

$$
\begin{aligned}
\operatorname{pre} \mathbb{E}\left(\mathrm{e}_{i}^{\prime}\right) & =\sum_{\tau \in \mathcal{T}} \mathbb{1}_{\mathbf{g}_{\tau} \geq 0} \times\left(\lambda^{T} \mathbf{e}+\mu_{\tau}^{T} \mathbf{g}_{\tau}\right) \\
& =\sum_{\tau \in \mathcal{T}} \mathbb{1}_{\mathbf{g}_{\tau} \geq 0} \times\left(\lambda^{T} \mathbf{e}\right)+\sum_{\tau \in \mathcal{T}} \mathbb{1}_{\mathbf{g}_{\tau} \geq 0} \times\left(\mu_{\tau}^{T} \mathbf{g}_{\tau}\right) \\
& =\lambda^{T} \sum_{\tau \in \mathcal{T}} \mathbb{1}_{\mathbf{g}_{\tau} \geq 0} \times \mathbf{e}+\sum_{\tau \in \mathcal{T}} \mathbb{1}_{\mathbf{g}_{\tau} \geq 0} \times\left(\mu_{\tau}^{T} \mathbf{g}_{\tau}\right) .
\end{aligned}
$$

In particular, we note that having a common set of multipliers $\lambda$ across transitions allows us to rewrite the summation $\sum_{\tau \in \mathcal{T}} \mathbb{1}_{\mathbf{g}_{\tau} \geq 0} \times\left(\lambda^{T} \mathbf{e}\right)$ as $\lambda^{T} \sum_{\tau \in \mathcal{T}} \mathbb{1}_{\mathbf{g}_{\tau} \geq 0} \times \mathbf{e}$. Next, since the transition guards are mutually exclusive and exhaustive, it follows that $\sum_{\tau \in \mathcal{T}} \mathbb{1}_{\mathbf{g}_{\tau} \geq 0} \times \mathbf{e}=\mathbf{e}$. Therefore, we write

$$
\operatorname{pre} \mathbb{E}\left(\mathrm{e}_{i}^{\prime}\right)=\lambda^{T} \mathbf{e}+\sum_{\tau \in \mathcal{T}} \mathbb{1}_{\mathbf{g}_{\tau} \geq 0} \times \mu_{\tau}^{T} \mathbf{g}_{\tau}
$$

This concludes the proof.

Theorem 3.3. Let $E$ be a conic inductive invariant for a program $\mathcal{P}$ as given by Definition 3.5. It follows that each $\mathrm{e}_{j} \in E$ is an expectation invariant of the program.

Proof. Proof simply combines Lemma 3.3 with Lemma 3.2 . 


\subsection{Pre-Expectation Closed Cones}

Thus far, we have presented inductive expectation invariants as a finite set of expressions $E=\left\{\mathrm{e}_{1}, \ldots, \mathrm{e}_{m}\right\}$, satisfying the conditions in Definition 3.4 or 3.5. We transfer our notion from a finite set of expressions to a finitely generated cone of these in preparation for our fixed point characterization in the next section.

Definition 3.6 (Cones). Let $E=\left\{\mathrm{e}_{1}, \ldots, \mathrm{e}_{k}\right\}$ be a finite set of program expressions over the program variables $\mathbf{x}$. The set of conic combinations (the cone) of $E$ is defined as

$$
\text { Cone }(E)=\left\{\lambda_{0}+\sum_{i=1}^{k} \lambda_{i} \mathrm{e}_{i} \mid 0 \leq \lambda_{i}, 0 \leq i \leq k\right\} .
$$

Expressions $\mathrm{e}_{i}$ are called the generators of the cone.

Given a non-empty linear assertion assertion $\varphi: \bigwedge_{i=1}^{k} \mathrm{e}_{i} \geq 0$, it is well-known that $\varphi \models \mathrm{e} \geq 0$ iff $\mathrm{e} \in \operatorname{Cone}\left(\mathrm{e}_{1}, \ldots, \mathrm{e}_{k}\right)$. Likewise, let $E$ be an inductive expectation invariant. It follows that any $\mathrm{e} \in \operatorname{Cone}(E)$ is an expectation invariant of the program $\mathcal{P}$.

Example 3.4. Revisiting Example 3.2, we consider the conic combination:

$$
4(-2 \mathrm{x}+\mathrm{y}-3)+3(4 \mathrm{x}-3 \mathrm{count}+4)=4 \mathrm{x}+4 \mathrm{y}-9 \text { count }
$$

As a result, we conclude that $\mathbb{E}_{\mathcal{D}_{n}}(4 \mathrm{x}+4 \mathrm{y}-9$ count $) \geq 0$ at each step $n \geq 0$.

Analyzing the program by replacing the probabilistic statements with non-deterministic choice, and performing polyhedral abstract interpretation yields the invariant $\mathrm{x}+\mathrm{y} \leq 14[8]$. This allows us to bound the set of support for $\mathcal{D}_{n}$, and also allows us to conclude that $\mathbb{E}_{\mathcal{D}_{n}}(14-\mathrm{x}-\mathrm{y}) \geq 0$. Combining these facts, we obtain,

$$
\mathbb{E}_{\mathcal{D}_{n}}(56-9 \text { count }) \geq 0, \text { or equivalently, } \mathbb{E}_{\mathcal{D}_{n}}(\text { count }) \leq \frac{56}{9}
$$

Conic Representations: A finitely generated cone Cone $\left(\mathrm{e}_{1}, \ldots, \mathrm{e}_{k}\right)$ of affine expressions $\mathrm{e}_{1}, \ldots, \mathrm{e}_{k}$ is represented using a polyhedral cone. Specifically, let e : $c_{0}+\mathbf{c}^{T} \mathbf{x}$ be any element of the cone. The polyhedral cone representation uses variables $\left(c_{0}, \mathbf{c}\right)$. Such a polyhedron can be represented using the constraint representation as:

$$
C: P\left(\begin{array}{c}
\mathbf{c} \\
c_{0}
\end{array}\right) \leq 0
$$

or as a set of generators given by the coefficient vectors of the expressions $e_{1}, \ldots, e_{k}$.

Example 3.5. Consider the cone generated by expressions

$$
\mathrm{e}_{1}:-2 x+y-3, \mathrm{e}_{2}: 4 x-3 z+4 \text {. }
$$

Any element of the cone can be written as $c_{0}+c_{1} x+c_{2} y+c_{3} z$ wherein the constraints:

$$
\left(\exists \lambda_{1}, \lambda_{2} \geq 0\right)\left[\begin{array}{c}
c_{0}=-3 \lambda_{1}+4 \lambda_{2} \wedge c_{1}=-2 \lambda_{1} \\
c_{2}=\lambda_{1} \wedge c_{3}=-3 \lambda_{2}
\end{array}\right]
$$

Alternatively, we may express the cone with a vertex $\left(c_{0}, c_{1}, c_{2}, c_{3}\right):(0,0,0,0)$ and rays:

$$
\left\{\left(\begin{array}{c}
-3 \\
-2 \\
1 \\
0
\end{array}\right),\left(\begin{array}{c}
4 \\
4 \\
0 \\
-3
\end{array}\right)\right\}
$$




\section{Expectation Invariants as Fixed Points}

In this section, we show that the notion of conic invariants as presented in Definition 3.5 can be expressed as a (pre-) fixed point of a monotone operator over finitely generated cones representing sets of expressions. This naturally allows us to use abstract interpretation starting from the cone representing all expressions $(T)$ and performing a downward Kleene iteration until convergence. We use a (dualized) widening operator to ensure fast convergence to fixed point in finitely many iterations.

Let $\mathcal{P}$ be a program over variables $\mathbf{x}$ with transitions $\mathcal{T}:\left\{\tau_{1}, \ldots, \tau_{k}\right\}$ and initial distribution $\mathcal{D}_{0}$. For simplicity, we describe our approach to generate affine expressions of the form $c_{0}+\mathbf{c}^{T} \mathbf{x}$ for $c_{0} \in \mathbb{R}, \mathbf{c} \in \mathbb{R}^{n}$. Let $\mathbb{A}(\mathbf{x})$ represent the set of all affine expressions over $\mathbf{x}$.

Polyhedral Cones of Expectation Invariant Candidates: Our approach uses finitely generated cones $I$ : Cone $(E)$ where $E=\left\{\mathrm{e}_{1}, \ldots, \mathrm{e}_{m}\right\}$ is a finite set of affine expressions over $\mathbf{x}$. Each element e $\in I$ represents a candidate expectation invariant. Once a (pre-) fixed point is found by our technique, we obtain a cone $I^{*}$ : $\operatorname{Cone}\left(E^{*}\right)$, wherein $E^{*}$ will be shown to be a conic inductive invariant according to Definition 3.5 .

A finitely generated cone of affine expressions $I$ : Cone $(E)$ is represented by a polyhedral cone of its coefficients $C(I):\left\{\left(c_{0}, \mathbf{c}\right) \mid c_{0}+\mathbf{c}^{T} \mathbf{x} \in I\right\}$. The generators of $C(I)$ are coefficient vectors $\left(c_{0, i}, \mathbf{c}_{i}\right)$ representing the expression $\mathrm{e}_{i}: c_{0, i}+\mathbf{c}_{i}^{T} \mathbf{x}$.

Our analysis operates on the lattice of polyhedral cone representations, Cones, ordered by the set theoretic inclusion operator $\subseteq$. This is, in fact, dual to the polyhedral domain, originally proposed by Cousot \& Halbwachs [8].

Initial Cone: For simplicity, we will assume that $\mathcal{D}_{0}$ is specified to us, and we are able to compute $E_{\mathcal{D}_{0}}(\mathbf{x})$ precisely for each program variable. The initial cone $I_{0}$ is given by

$$
I_{0}: \text { Cone }\left(\left\{x_{1}-\mathbb{E}_{\mathcal{D}_{0}}\left(x_{1}\right), \mathbb{E}_{\mathcal{D}_{0}}\left(x_{1}\right)-x_{1}, \cdots, \mathbb{E}_{\mathcal{D}_{0}}\left(x_{n}\right)-x_{n}, x_{n}-\mathbb{E}_{\mathcal{D}_{0}}\left(x_{n}\right)\right\}\right) .
$$

Such a cone represents the invariant candidates $x_{i}=\mathbb{E}_{\mathcal{D}_{0}}\left(x_{i}\right)$. The representation of the initial cone is given by the set of $2 n$ rays of the form $\left[\mathbb{E}_{\mathcal{D}_{0}}\left(x_{i}\right) \quad 0 \cdots \cdots\right.$

Pre-Expectation Operators: We now describe the parts of the monotone operator over finitely generated cones. Let $E=\left\{\mathrm{e}_{1}, \ldots, \mathrm{e}_{m}\right\}$ be a set of expressions. Let $\tau:\langle\mathrm{g}, \mathcal{F}\rangle$ be a transition, wherein $\mathrm{g}: \bigwedge_{l=1}^{p} g_{l} \geq 0$. We first present a pre-expectation operator over cones, lifting the notation pre $\mathbb{E}_{\tau}$ from expressions to cones of such:

Definition 4.1 (Pre-Expectation Operator). The pre-expectation of a cone $I$ : Cone $(E)$ w.r.t a transition $\tau$ is defined as:

$$
\operatorname{pre} \mathbb{E}_{\tau}(I)=\left\{(\mathrm{e}, \lambda) \in \mathbb{A}(x) \times \mathbb{R}^{m} \mid \lambda \geq 0 \wedge \exists \mu \geq 0\left(\operatorname{pre}_{\tau}\left(\mathrm{e}^{\prime}\right) \equiv \sum_{j=1}^{m} \lambda_{j} \mathrm{e}_{j}+\sum_{i=1}^{p} \mu_{i} \mathbf{g}_{i}\right)\right\} .
$$

The refinement $\operatorname{pre} \mathbb{E}_{\tau}(I)$ of a cone contains all affine program expressions whose pre-expectation belongs to the conic hull of $I$ and the cone generated by the guard assertion. For technical reasons, we attach to each expression a certificate $\lambda$ that shows its membership back in the cone. This can be seen as a way to ensure the proper order of quantification in Definition 3.5 .

Given a polyhedron $C(I)$ representing $I$, we can show that $C\left(\operatorname{pre} \mathbb{E}_{\tau}(I)\right)$ is a polyhedral cone over the variables $\left(c_{0}, \mathbf{c}\right)$ representing the expression coefficients and $\lambda$ for the multipliers.

Lemma 4.1. For a given cone $C$, the pre-expectation operator across a transition pre $\mathbb{E}_{\tau}(C)$ is also a cone.

PreExpectation of Cones: First, we define the lifting of pre $\mathbb{E}_{\tau}(I)$ for a single cone of expressions $I$. Let $\tau$ be given by the guard set $\bigwedge_{i=1}^{l} \mathbf{g}_{i}^{T} \mathbf{x}+h_{i} \geq 0$, and update with forks $f_{1}, \ldots, f_{k}$ wherein $f_{i}: A_{i} \mathbf{x}+B_{i} \mathbf{r}+\mathbf{a}_{i}$ 
is taken with probability $p_{i}$. Consider a generic next state affine expression: e : $c_{0}+\mathbf{c}^{T} \mathbf{x}^{\prime}$. We write

$$
\operatorname{pre} \mathbb{E}_{\tau}\left(\mathrm{e}^{\prime}\right): c_{0}+\mathbf{c}^{T}\left(p_{1} A_{1}+\cdots+p_{k} A_{k}\right) \mathbf{x}+\mathbf{c}^{T} \mathbb{E}_{\mathcal{D}_{R}}\left(p_{1} B_{1} \mathbf{r}+\cdots+p_{k} B_{k} \mathbf{r}\right)+\mathbf{c}^{T}\left(p_{1} \mathbf{a}_{1}+\cdots+p_{k} \mathbf{a}_{k}\right) .
$$

Simplifying, we write

$$
\operatorname{pre} \mathbb{E}_{\tau}\left(\mathrm{e}^{\prime}\right):\left(c_{0}+\alpha^{T} \mathbf{c}\right)+\mathbf{c}^{T} \mathcal{B} \mathbf{x} .
$$

Let $I$ be the cone generated by the expressions $\left\{e_{1}, \ldots, e_{k}\right\}$ wherein $e_{j}: d_{0, j}+\mathbf{d}_{j}^{T} \mathbf{x}$. The generators of the cone are given by the rays $\left(d_{0, j}, \mathbf{d}_{j}\right)$ for $j=1, \ldots, k$. We compute an augmented representation of the cone $I$ given by the constraints:

$$
P_{I}\left(d_{0}, \mathbf{d}, \lambda, \mu\right): \lambda \geq 0 \wedge \mu \geq 0 \wedge d_{0}=\sum_{j=1}^{k} \lambda_{j} d_{0, j}+\sum_{i=1}^{l} \mu_{i} h_{i} \wedge \mathbf{d}=\sum_{j=1}^{k} \lambda_{j} \mathbf{d}_{j}+\sum_{i=1}^{l} \mu_{i} \mathbf{g}_{i} .
$$

The polyhedron $P_{I}$ represents all combinations of expressions $\left(d_{0}+\mathbf{d}^{T} \mathbf{x}\right)$ that are derived by a conic combination of expressions $\mathrm{e}_{1}, \ldots, \mathrm{e}_{k}$ through multipliers $\lambda_{1}, \ldots, \lambda_{k} \geq 0$ and guard inequality expressions $\mathbf{g}_{1}, \ldots, \mathbf{g}_{l}$ through multipliers $\mu_{1}, \ldots, \mu_{l} \geq 0$. The cone $\operatorname{pre} \mathbb{E}_{\tau}(I)$ is given as

$$
\operatorname{pre} \mathbb{E}_{\tau}(I):(\exists \mu) P_{I}\left(c_{0}+\alpha^{T} \mathbf{c}, \mathcal{B}^{T} \mathbf{c}, \lambda, \mu\right) .
$$

Note that $\operatorname{pre} \mathbb{E}_{\tau}(I)$ is a polyhedron over variables $\left(c_{0}, \mathbf{c}\right)$ representing an expression e : $c_{0}+\mathbf{c}^{T} \mathbf{x}$ and multipliers $\lambda \in \mathbb{R}^{k}$.

Next, we define a pre-expectation operator across all transitions:

$$
\operatorname{pre} \mathbb{E}(I)=\left\{\mathrm{e} \in \mathbb{A}(x) \mid(\exists \lambda \geq 0)(\mathrm{e}, \lambda) \in \bigcap_{j=1}^{k} \operatorname{pre}_{\tau_{j}}(I)\right\}
$$

An expression e belongs to pre $\mathbb{E}(I)$ if for some $\lambda \geq 0,(e, \lambda) \in \operatorname{pre} \mathbb{E}_{\tau_{j}}(I)$ for each transition $\tau_{j} \in \mathcal{T}$.

Given a cone $C(I)$, we first compute the cones $C\left(\hat{I}_{1}\right), \ldots, C\left(\hat{I}_{k}\right)$ representing the pre-expectations across transitions $\tau_{1}, \ldots, \tau_{k}$, respectively. Next, we compute $C\left(I^{\prime}\right):(\exists \lambda) \bigcap_{j=1}^{k} C\left(\hat{I}_{j}\right)$, representing $I^{\prime}$ : pre $\mathbb{E}(I)$, by intersecting the cones $C\left(\hat{I}_{j}\right)$ and projecting the dimensions corresponding to $\lambda$.

We define the operator $\mathcal{G}$ over cones as $\mathcal{G}(I): I_{0} \cap$ pre $\mathbb{E}(I)$, where $I_{0}$ is the initial cone.

Theorem 4.1. The operator $\mathcal{G}$ satisfies the following properties:

1. $\mathcal{G}$ is a monotone operator over the lattice CONES ordered by set-theoretic inclusion.

2. A finite set of affine expressions $E$ is a conic inductive invariant (Definition 3.5) iff I : Cone $(E)$ is a pre-fixed point of $\mathcal{G}$, i.e, $I \subseteq \mathcal{G}(I)$.

\subsection{Proof of Theorem 4.1}

The details of the proof are quite intricate, so we build the proof of Theorem 4.1 in a bottom up fashion.

Let $\mathcal{P}:\left\langle\mathcal{T}, \mathcal{D}_{0}, n\right\rangle$ be a probabilistic loop with $\mathcal{T}=\left\{\tau_{1}, \ldots, \tau_{m}\right\}$, where each transition is of the form $\tau_{i}:\left\langle\mathbf{g}_{i}, \mathcal{F}_{i}\right\rangle$. We begin by showing that pre $\mathbb{E}_{\tau}$ is a monotone operator.

Lemma 4.2. Let $I_{1}=$ Cone $\left(e_{1}, \ldots, e_{m}\right)$ and $I_{2}=$ Cone $\left(h_{1}, \ldots, h_{k}\right)$ be two finitely generated cones such that $I_{1} \subseteq I_{2}$ and let $\tau_{i} \in \mathcal{T}$, then $\operatorname{pre} \mathbb{E}_{\tau_{i}}\left(I_{1}\right) \subseteq \operatorname{pre} \mathbb{E}_{\tau_{i}}\left(I_{2}\right)$. 
Proof. Let $(\mathrm{e}, \lambda) \in \operatorname{pre}_{\tau_{i}}\left(I_{1}\right)$ for some e $\in \mathbb{A}(x)$ and $\lambda \geq 0$. By Definition 4 , there exists $\mu_{i} \geq 0$ such that $\operatorname{pre} \mathbb{E}_{\tau_{i}}(\mathrm{e})=\lambda^{T}\left(e_{1} \cdots e_{j}\right)^{T}+\mu_{i}^{T} \mathbf{g}_{i}$.

Since $I_{1} \subseteq I_{2}$ then for every generator $e_{j}$ of $I_{1}$ there exist non-negative coefficients $\lambda_{j 1}, \ldots, \lambda_{j k}$ such that $e_{j}=\lambda_{j}^{T}\left(h_{1} \cdots h_{k}\right)$. Therefore, we can define the change of basis transformation matrix $\Lambda=\left[\begin{array}{lll}\lambda_{11} & \cdots & \lambda_{1 k} \\ \vdots & \ddots & \vdots \\ \lambda_{m 1} & \cdots & \lambda_{m k}\end{array}\right]$ such that $\left[\begin{array}{l}e_{1} \\ \vdots \\ e_{m}\end{array}\right]=\Lambda\left[\begin{array}{l}h_{1} \\ \vdots \\ h_{k}\end{array}\right]$. Notice that $\Lambda$ is independent of $\tau_{i}$; moreover, $\Lambda$ is a non-negative matrix.

This means that pre $\mathbb{E}_{\tau_{i}}(\mathrm{e})=\lambda^{T}\left(e_{1} \cdots e_{m}\right)^{T}+\mu_{i}^{T} \mathbf{g}_{i}=\lambda^{T} \Lambda\left(h_{1} \cdots h_{k}\right)^{T}+\mu_{i}^{T} \mathbf{g}_{i}$. Therefore, there exists a non-negative $\lambda^{\prime} \equiv \lambda^{T} \Lambda$ such that $\left(\mathrm{e}, \lambda^{\prime}\right)$ belongs to $I_{2}$.

A consequence of Lemma 4.2 we see that for every $(\mathrm{e}, \lambda)$ pair in $I_{1}$, there exists a unique $\left(\mathrm{e}, \lambda^{\prime}\right)$ pair in $I_{2}$ regardless of the guard $\mathbf{g}_{i}$ (and therefore, transition $\tau_{i}$ ). The following result follows immediately.

Lemma 4.3 (Monotonicity of pre $\mathbb{E})$. Let $I_{1}=$ Cone $\left(e_{1}, \ldots, e_{m}\right)$ and $I_{2}=$ Cone $\left(h_{1}, \ldots, h_{k}\right)$ such that $I_{1} \subseteq I_{2}$, then pre $\mathbb{E}\left(I_{1}\right) \subseteq \operatorname{pre} \mathbb{E}\left(I_{2}\right)$.

Proof. If $\mathbf{e} \in \operatorname{pre} \mathbb{E}\left(I_{1}\right)$ then there exists a signature $\lambda \geq 0$ such that $(\mathrm{e}, \lambda) \in \bigcap_{\tau \in \mathcal{T}} \operatorname{pre} \mathbb{E}_{\tau}\left(I_{1}\right)$. Define $\lambda^{\prime} \equiv \lambda^{T} \Lambda$. Then $\left(\mathrm{e}, \lambda^{\prime}\right)$ belongs to $\bigcap_{\tau \in \mathcal{T}} \operatorname{pre}_{\mathcal{\tau}}\left(I_{2}\right)$. Therefore, e belongs to pre $\mathbb{E}\left(I_{2}\right)$. This completes the proof of monotonicity.

Lemma 4.4. Let $E$ be a conic inductive invariant, then $C o n e(E) \subseteq I_{0}$.

Proof. WLOG, let $\mathrm{e}_{i} \in E$, then by construction, $\mathrm{e}_{i}$ is a generator of $C o n e(E)$. By Definition 3.5, we know that $\mathbb{E}_{\mathcal{D}_{0}}\left(\mathrm{e}_{i}\right)=k$, for some $k \geq 0$. On the other hand, $\mathrm{e}_{i}$ is a linear expression, so $\mathrm{e}_{i}: c_{0}+\mathbf{c}^{T} \mathbf{x}$ for some $c_{0} \geq 0, \mathbf{c} \geq 0$.

$$
I_{0} \equiv \operatorname{Cone}\left(\left\{1, x_{1}-\mathbb{E}_{\mathcal{D}_{0}}\left(x_{1}\right), \mathbb{E}_{\mathcal{D}_{0}}\left(x_{1}\right)-x_{1}, \cdots, x_{n}-\mathbb{E}_{\mathcal{D}_{0}}\left(x_{n}\right), \mathbb{E}_{\mathcal{D}_{0}}\left(x_{n}\right)-x_{n}\right\}\right) .
$$

For every $j \geq 1$, define $\left(\lambda_{2 j-1}, \lambda_{2 j}\right) \equiv\left(c_{j}, 0\right)$ if $c_{j} \geq 0$ and $\left(0,-c_{j}\right)$ otherwise. Finally, define $\lambda_{0} \equiv k-\sum c_{j}$. It then follows that $\mathrm{e}_{i}=\lambda^{T} \mathbf{i}_{\mathbf{0}}$ with $\lambda=\left(\lambda_{0}, \lambda_{1}, \cdots, \lambda_{2 n}\right) \geq 0$.

Therefore, $\mathrm{e}_{i} \in I_{0}$.

Lemma 4.5. Let $E$ be a conic inductive invariant and $I=C o n e(E)$, then $I \subseteq \operatorname{pre} \mathbb{E}(I)$.

Proof. Let e $\in I$, then by Definition 3.5, there exists a certificate $\lambda \geq 0$ that satisfies the requirements of Definition 4, simultaneously, for every transition.

Therefore, e $\in \operatorname{pre} \mathbb{E}(I)$.

Theorem 4.2 (Theorem 4.1). The operator $\mathcal{G}$ satisfies the following properties:

1. $\mathcal{G}$ is a monotone operator over the lattice CONES ordered by set-theoretic inclusion.

2. A finite set of affine expressions $E$ is a conic inductive invariant (Def. 3.5) if and only if $I$ : Cone $(E)$ is a pre-fixed point of $\mathcal{G}$, i.e, $I \subseteq \mathcal{G}(I)$.

Proof. Part 1: Let $I_{1}=$ Cone $\left(e_{1}, \ldots, e_{m}\right)$ and $I_{2}=$ Cone $\left(h_{1}, \ldots, h_{k}\right)$ such that $I_{1} \subseteq I_{2}$. Expanding the definition of $\mathcal{G}$ and applying Lemma $4.3, \mathcal{G}\left(I_{1}\right)=I_{0} \cap \operatorname{pre} \mathbb{E}\left(I_{1}\right) \subseteq I_{0} \cap \operatorname{pre} \mathbb{E}\left(I_{2}\right)=\mathcal{G}\left(I_{2}\right)$. This completes the proof that if $I_{1} \subseteq I_{2}$ then $\mathcal{G}\left(I_{1}\right) \subseteq \mathcal{G}\left(I_{2}\right)$.

Part $2(\Rightarrow)$ : Let $E$ be a conic inductive expectation invariant, then by Lemma 4.4, $I=C o n e(E)$ is a subset of $I_{0}$. By Lemma 4.5, we know $I \subseteq \operatorname{pre} \mathbb{E}(I)$. Therefore, by definition of $\mathcal{G}, I$ is a pre-fixed point of $\mathcal{G}$.

$(\Leftarrow)$ : Let $I=C o n e(E)$ for some set of expressions $E$ such that $I \subseteq \mathcal{G}(I)$. Then $I \subseteq I_{0} \cap \operatorname{pre} \mathbb{E}(I)$. Since $I \subseteq \operatorname{pre} \mathbb{E}(I)$ then there exists a certificate $\lambda \geq 0$ common for all transitions $\tau$; this satisfies condition (2) of 
Definition 3.5. Now let e : $c_{0}+\mathbf{c}^{T} \mathbf{x}$ be a linear expression in $I$. Since $I \subseteq I_{0}$, then e : $\lambda_{0}^{\prime}+\sum_{i=1}^{m}\left[\lambda_{2 i-1}^{\prime}\left(x_{i}-\right.\right.$ $\left.\left.\mathbb{E}_{\mathcal{D}_{0}}\left(x_{i}\right)\right)+\lambda_{2 i}^{\prime}\left(\mathbb{E}_{\mathcal{D}_{0}}\left(x_{i}\right)-x_{i}\right)\right]=\lambda_{0}^{\prime}+\sum_{i=1}^{m}\left[\left(\lambda_{2 i-1}^{\prime}-\lambda_{2 i}^{\prime}\right) x_{i}+\left(\lambda_{2 i}^{\prime}-\lambda_{2 i-1}^{\prime}\right) \mathbb{E}_{\mathcal{D}_{0}}\left(x_{i}\right)\right]$ for some non-negative scalars $\lambda_{j}^{\prime}$. Define $\kappa_{i} \equiv \lambda_{2 i-1}^{\prime}-\lambda_{2 i}^{\prime}$. The expectation $\mathbb{E}_{\mathcal{D}_{0}}(\mathrm{e})=\mathbb{E}_{\mathcal{D}_{0}}\left(\lambda_{0}^{\prime}+\sum_{i=1}^{m}\left[\kappa_{i} x_{i}-\kappa_{i} \mathbb{E}_{\mathcal{D}_{0}}\left(x_{i}\right)\right]\right) \geq 0$. Therefore, e satisfies Definition 3.5(1). Thus, $E$ is a conic inductive expectation invariant.

\subsection{Iteration over Polyhedral Cones}

Our goal is to compute the greatest fixed point of $\mathcal{G}$ representing the largest cone of expressions whose generators satisfy Definition 3.5. We implement this by a downward Kleene iteration until we obtain a pre-fixed point, which in the ideal case is also the greatest fixed point of $\mathcal{G}$.

$$
\left(J_{0}: \mathbb{A}(x)\right) \supseteq\left(J_{1}: \mathcal{G}\left(J_{0}\right)\right) \supseteq \cdots\left(J_{k+1}: \mathcal{G}\left(J_{k}\right)\right) \cdots \text { until convergence: } J_{i} \subseteq J_{i+1} .
$$

However, the domain Cones has infinite descending chains and is not a complete lattice. Therefore, the greatest fixed point cannot necessarily be found in finitely many steps by the Kleene iteration. We resort to a dual widening operator $\widetilde{\nabla}$ to force convergence of the downward iteration.

Definition 4.2 (Dual Widening). Let $I_{1}, I_{2}$ be two successive cone iterates, satisfying $I_{1} \supseteq I_{2}$. The operator $\widetilde{\nabla}\left(I_{1}, I_{2}\right)$ is a dual widening operator if:

- $\widetilde{\nabla}\left(I_{1}, I_{2}\right) \subseteq I_{1}, \widetilde{\nabla}\left(I_{1}, I_{2}\right) \subseteq I_{2}$

- For every infinite descending sequence $J_{0} \supseteq \mathcal{G}\left(J_{0}\right) \supseteq \mathcal{G}^{2}\left(J_{0}\right) \supseteq \cdots$, the widened sequence $J_{0}^{\prime}=J_{0}$, $J_{n}^{\prime}=J_{n-1}^{\prime} \widetilde{\nabla} J_{n}$ converges in finitely many steps.

A common strategy to compute an approximation of the greatest fixed point when using dual widening is to delay widening for a fixed number $K$ of iterations.

Example 4.1. Consider a simulation of a peg performing an unbounded random walk in two dimensions $(\mathrm{x}, \mathrm{y})$. Starting at the origin, at every step the peg chooses uniformly at random a direction $\{\mathrm{N}, \mathrm{E}, \mathrm{S}, \mathrm{W}\}$ and a random step size $r_{1} \sim U[0,2]$. The program 2D-WALK tracks the steps (count) and the Manhattan distance (dist) to the origin.

The following table summarizes the result of the expectation invariant analysis:

\begin{tabular}{|c|c|c|c|c|c|}
\hline Cone & Generators & Constraints & Cone & Generators & Constraints \\
\hline$I_{0}$ & $\begin{array}{c}1,- \text { count, count, } \\
\mathrm{x},-\mathrm{x}, \mathrm{y},-\mathrm{y}, \text { dist, }- \text { dist, }\end{array}$ & $c_{0} \geq 0$ & $I_{4}$ & $\begin{array}{c}1,4-\text { count, count, } \\
\mathrm{x},-\mathrm{x}, \mathrm{y},-\mathrm{y}, \text { dist, }- \text { dist }\end{array}$ & $\begin{array}{c}c_{0}+4 c_{4} \geq 0 \\
c_{0} \geq 0\end{array}$ \\
\hline$I_{1}$ & $\begin{array}{c}1,1-\text { count, count, } \\
\mathrm{x},-\mathrm{x}, \mathrm{y},-\mathrm{y}, \text { dist, }- \text { dist }\end{array}$ & $\begin{array}{c}c_{0}+c_{4} \geq 0 \\
c_{0} \geq 0\end{array}$ & $I_{5}$ & $\begin{array}{c}1,5-\text { count, count, } \\
\mathrm{x},-\mathrm{x}, \mathrm{y},-\mathrm{y}, \text { dist, }- \text { dist }\end{array}$ & $\begin{array}{c}c_{0}+4 c_{4} \geq 0 \\
c_{0} \geq 0\end{array}$ \\
\hline$I_{2}$ & $\begin{array}{c}1,2-\text { count, count, } \\
\mathrm{x},-\mathrm{x}, \mathrm{y},-\mathrm{y}, \text { dist, }- \text { dist }\end{array}$ & $\begin{array}{c}c_{0}+2 c_{4} \geq 0 \\
c_{0} \geq 0\end{array}$ & : & 政 & \\
\hline$I_{3}$ & $\begin{array}{c}1,3-\text { count, count, } \\
\mathrm{x},-\mathrm{x}, \mathrm{y},-\mathrm{y}, \text { dist, }- \text { dist }\end{array}$ & $\begin{array}{c}c_{0}+3 c_{4} \geq 0 \\
c_{0} \geq 0\end{array}$ & $I_{\infty}$ & $\begin{array}{c}1, \text { count, } \\
\mathrm{x},-\mathrm{x}, \mathrm{y},-\mathrm{y}, \text { dist, }- \text { dist }\end{array}$ & $\begin{array}{l}c_{4} \geq 0 \\
c_{0} \geq 0\end{array}$ \\
\hline
\end{tabular}

The table shows the value of expression count is unbounded from above. To force convergence, we employ dual widening after a predefined number $(K=5)$ of iterations.

Definition 4.3 (Standard Dual Widening). Let $I_{1}=C o n e\left(g_{1}, \ldots, g_{k}\right)$ and $I_{2}=C o n e\left(h_{1}, \ldots, h_{l}\right)$ be two finitely generated cones such that $I_{1} \supseteq I_{2}$. The dual widening operator $I_{1} \widetilde{\nabla} I_{2}$ is defined as $I=$ Cone $\left(g_{i} \mid g_{i} \in I_{2}\right)$. Cone $I$ is the cone generated by the generators of $I_{1}$ that are subsumed by $I_{2}$.

Example 4.2. Returning to Example 4.1, we consider cone iterates $I_{4}, I_{5}$. In this case generator subsumption reduces to a simple containment check. Since generator $\mathbf{4}$ - count is not subsumed in $I_{5}$, we arrive at $I_{5}^{\prime} \equiv I_{4} \widetilde{\nabla} I_{5}=\widetilde{I^{*}}=I_{\infty}$.

Note 1. Alternatively, one can define dual widening as a widening operator [12, 1] over the dual polyhedron that the generators of $I_{1}, I_{2}$ give rise to. On the set of PWL loop benchmarks our dual widening approach and those based on [12] and [1] produce identical fixed points where the difference in timings is not statistically significant. 
Table 1: Summary of results: $|X|$ is the number of program variables; $|\mathcal{T}|$ - transitions; \# - iterations to convergence; $\widetilde{\nabla}$ - use of dual widening. Lines (Rays) is the number of resultant inductive expectation equalities (inequalities). Time is taken on a MacBook Pro $(2.4 \mathrm{GHz})$ laptop with $8 \mathrm{~GB}$ RAM, running MacOS X 10.9.1 (where $\varepsilon=0.05 \mathrm{sec}$ ).

\begin{tabular}{|c|c|c|c|c|c|c|c|c|}
\hline \multirow{2}{*}{ Name } & \multirow{2}{*}{ Description } & \multirow{2}{*}{$|X|$} & \multirow{2}{*}{$|\mathcal{T}|$} & \multicolumn{2}{|c|}{ Iters } & \multicolumn{2}{|c|}{ Fixpoint-gen } & \multirow{2}{*}{ Time } \\
\hline & & & & $\#$ & $\widetilde{\nabla}$ & Lines & Rays & \\
\hline MOT-EXAMPLE & Motivating Example of Figure 1 & 3 & 2 & 2 & No & 2 & 1 & $\leq \varepsilon$ \\
\hline MOT-EX-LOOP-INV & Example 1.1 with added loop invariants & 3 & 2 & 2 & No & 2 & 2 & 0.10 \\
\hline MOT-EX-POLY & Ex. 1.1 generate poly constr $(\operatorname{deg} \leq 2)$ & 9 & 2 & 2 & No & 5 & 2 & 0.18 \\
\hline 2D-WALK & Random walk in 2 dimensions & 4 & 4 & 7 & Yes & 3 & 1 & $\leq \varepsilon$ \\
\hline AGGREGATE-RV & Accumulate RVs & 3 & 2 & 2 & No & 2 & 0 & $\leq \varepsilon$ \\
\hline HARE-TURTLE & Stochastic Hare-Turtle race & 3 & 2 & 2 & No & 1 & 1 & $\leq \varepsilon$ \\
\hline COUPON5 & Coupon Collector's Problem $(\mathrm{n}=5)$ & 2 & 5 & 2 & No & 1 & 2 & $\leq \varepsilon$ \\
\hline FAIR-COIN-BIASED & Simulating biased coin with fair coin & 3 & 2 & 3 & No & 1 & 1 & $\leq \varepsilon$ \\
\hline HAWK-DOVE-FAIR & Stochastic 2-player game (collaborate) & 6 & 2 & 2 & No & 4 & 1 & $\leq \varepsilon$ \\
\hline HAWK-DOVE-BIAS & Stochastic 2-player game (exploit) & 6 & 2 & 2 & No & 3 & 1 & $\leq \varepsilon$ \\
\hline FAULTY-INCR & Faulty incrementor & 2 & 2 & 7 & Yes & 1 & 1 & $\leq \varepsilon$ \\
\hline
\end{tabular}

\section{Experimental Results and Future Work}

We present the experimental results of our prototype implementation that relies on PPL [1] for manipulating the polyhedral representations of cones. Table 1 presents the summary of the experiments we conducted on a set of probabilistic benchmarks. In [4], we present a description of these models and the expectation invariants obtained.

In all experiments we emphasize precision over computational effort. All examples except MOT-EXLOOP-INV and MOT-EX-POLY run in under $\varepsilon=0.05$ seconds, so we choose not to report these timing. Accordingly, dual widening $\widetilde{\nabla}$ delay was set sufficiently large at $K=5$ to only force finite convergence but not to speed up computation. Nevertheless, the iterations converge quite fast and in many cases without the use of widening. Programs 2D-WALK and FAULTY-INCR require the widening $(\widetilde{\nabla})$ operator to ensure convergence. In all cases, line generators of the final pre-fixed point yield expectation invariants like $\mathbb{E}(\mathrm{e})=0$ and rays yield the invariants $\mathbb{E}(\mathrm{e}) \geq 0$.

\subsubsection{Comparison with PRINSYS[1].}

PRINSYS[11] implements the constraint-based quantitative invariant synthesis approach developed by Katoen et al. [14]. The tool uses a manually supplied template with unknown coefficients. The REDUCE computer algebra system is used to perform quantifier elimination and simplify the constraints. We applied PRINSYS with a linear template expression $\sum_{j} c_{j} x_{j}$ for all state variables $x_{j}$ in the program. Our comparison was carried out over the 6 benchmark examples distributed with the tool. The comparison checked whether PRINSYS could discover quantitative invariants discovered by our approach. Table 2 presents a summary of the comparison.

From a total set of 26 inductive expectation invariants our tool generates, PRINSYS could generate 3 of them. Notice that we had to manually provide some additional initial invariants which PRINSYS was able to trivially, yet correctly prove invariant (denoted by asterisk in last column). Overall, we observe that mutual inductive expectation invariants investigated in this paper provide interesting, significant facts about the probabilistic loops in the PRINSYS benchmarks.

Next, we attempted to check whether PRINSYS can discover additional linear quantitative invariants not discovered by our approach due to the incompleteness of widening. Unfortunately, this check turned out inconclusive at the time of the experiment. The existing PRINSYS implementation automatically generates and simplifies nonlinear constraints on the template coefficients. However, the process of deriving an actual quantitative invariant requires manually extracting solutions from a set of nonlinear 
Table 2: Summary of comparison results: IEI - invariants generated by our tool; Iters, Time - number of iterations, time for our tool to converge; PRINSYS - was PRINSYS able to infer this quantitative invariant.

\begin{tabular}{|c|c|c|c|c|}
\hline Name & IEI & Iters & Time & PRINSYS \\
\hline BIASED-COIN & $\begin{array}{c}2-2 b-\text { count }=0 \\
3-4 x \geq 0\end{array}$ & 6 & 0.0877 & $\begin{array}{l}\text { No } \\
\text { No }\end{array}$ \\
\hline BINOMIAL-UPDATE $(\mathrm{M}=20)$ & $\begin{array}{c}4 \mathrm{x}-3 \mathrm{n}=0 \\
\mathrm{x} \geq 0\end{array}$ & 21 & 0.1337 & $\begin{array}{l}\text { No } \\
\text { No }\end{array}$ \\
\hline COWBOYS & $\begin{array}{c}1-7 \text { turn }- \text { continue } \geq 0 \\
6-2 \text { turn }-6 \text { continue }-5 \text { count }=0\end{array}$ & 4 & 0.1146 & $\begin{array}{l}\text { No } \\
\text { No }\end{array}$ \\
\hline FAIR-COIN & $\begin{array}{c}x-y=0 \\
3-4 x \geq 0 \\
-4 x+3 \text { count } \geq 0\end{array}$ & 6 & 0.0844 & $\begin{array}{l}\text { Yes } \\
\text { No } \\
\text { No }\end{array}$ \\
\hline GEOMETRIC & $\begin{array}{c}x \geq 0 \\
3 x-\text { flip }=0 \\
4 x-\text { count }=0 \\
\text { count } \geq 0\end{array}$ & 29 & 0.1988 & $\begin{array}{l}\text { No } \\
\text { No } \\
\text { No } \\
\text { Yes* }\end{array}$ \\
\hline UNLIMITED-MARTINGALE & $\begin{array}{c}\text { rounds } \geq 0 \\
32 \mathrm{c}+\text { rounds } \leq 1600 \\
\mathrm{c}+\mathrm{b}=51 \\
(10 \text { additional inequalities })\end{array}$ & 13 & 0.2 & $\begin{array}{l}\text { Yes* } \\
\text { No } \\
\text { No } \\
\text { No }\end{array}$ \\
\hline
\end{tabular}

inequalities. Our manual efforts failed to find new invariants unique to the PRINSYS tool, but the overall comparison remains incomplete since we could not arguably find all solutions manually.

Finally, it is important to observe that PRINSYS can generate invariants for templates that include indicator functions, while our technique curently does not. Similarly, PRINSYS handles nondeterminism in the programs, while we do not.

Ongoing/Future Work. In many of the benchmark examples we present, we found that invariants discovered using standard abstract interpretation by treating the stochastic choices as demonic nondeterminism help improve the quality of our expectation invariants. Going further, we would like to combine classical abstract interpretation with the techniques presented here to handle programs that mix nondeterministic and stochastic choices. Finally, we demonstrate polynomial invariant synthesis in Example MOT-EX-POLY by instrumenting monomials of fixed degree $(\operatorname{deg} \leq 2)$ as fresh variables. Our analysis is thus able to generate polynomial expectation invariants such as $\mathbb{E}\left(4 x^{2}-4 x y+y^{2} \mid n\right) \geq 0$, and $\mathbb{E}\left(4 x^{2}-4 x y+y^{2}-y+6 \mid n\right)=0$. A sound formalization of polynomial invariant generation under relaxed independence conditions, and generalization of this approach to higher-order moments are also part of our future work.

Acknowledgments. The authors thank the anonymous reviewers for their insightful comments and Friedrich Gretz for helping us compare our work with PRINSYS. This work was supported by US National Science Foundation (NSF) under award number 1320069. All opinions are those of the authors and not necessarily of the NSF.

\section{References}

[1] R. Bagnara, P. M. Hill, E. Ricci, and E. Zaffanella. Precise widening operators for convex polyhedra. In Static Analysis, pages 337-354. Springer, 2003.

[2] O. Bouissou, E. Goubault, J. Goubault-Larrecq, and S. Putot. A generalization of p-boxes to affine arithmetic. Computing, 94(2-4):189-201, 2012. 
[3] A. Chakarov and S. Sankaranarayanan. Probabilistic program analysis with martingales. In $C A V$, pages 511-526, 2013.

[4] A. Chakarov and S. Sankaranarayanan. Expectation invaraiants for probabilistic program loops as fixed points (extended version), 2014. Draft, Available upon request.

[5] K. L. Chung. A course in probability theory, volume 3. Academic press New York, 1974.

[6] M. Colón, S. Sankaranarayanan, and H. Sipma. Linear invariant generation using non-linear constraint solving. In $C A V$, volume 2725 of $L N C S$, pages 420-433. Springer, July 2003.

[7] P. Cousot and R. Cousot. Abstract Interpretation: A unified lattice model for static analysis of programs by construction or approximation of fixpoints. In ACM Principles of Programming Languages, pages 238-252, 1977.

[8] P. Cousot and N. Halbwachs. Automatic discovery of linear restraints among the variables of a program. In POPL'78, pages 84-97, Jan. 1978.

[9] P. Cousot and M. Monerau. Probabilistic abstract interpretation. In H. Seidel, editor, 22nd European Symposium on Programming (ESOP 2012), volume 7211 of Lecture Notes in Computer Science, pages 166-190, Heidelberg, 2012. Springer-Verlag.

[10] D. Dubhashi and A. Panconesi. Concentration of Measure for the Analysis of Randomized Algorithms. Cambridge University Press, 2009.

[11] F. Gretz, J.-P. Katoen, and A. McIver. Prinsys - on a quest for probabilistic loop invariants. In QEST, pages 193-208, 2013.

[12] N. Halbwachs. Détermination automatique de relations linéaires vérifiées par les variables d'un programme. PhD thesis, Institut National Polytechnique de Grenoble-INPG, 1979.

[13] J.-P. Katoen, A. McIver, L. Meinicke, and C. Morgan. Linear-invariant generation for probabilistic programs. In Static Analysis Symposium (SAS), volume 6337 of LNCS, page 390406. Springer, 2010.

[14] J.-P. Katoen, A. K. McIver, L. A. Meinicke, and C. C. Morgan. Linear-invariant generation for probabilistic programs. In Static Analysis, pages 390-406. Springer, 2011.

[15] D. Kozen. Semantics of probabilistic programs. J. Comput. Syst. Sci., 22(3):328-350, 1981.

[16] Z. Manna and A. Pnueli. Temporal Verification of Reactive Systems: Safety. Springer, New York, 1995.

[17] P. Mardziel, S. Magill, M. Hicks, and M. Srivatsa. Dynamic enforcement of knowledge-based security policies. In Computer Security Foundations Symposium (CSF), 2011 IEEE 24th, pages 114-128. IEEE, 2011.

[18] H. McAdams and A. Arkin. It's a noisy business! genetic regulation at the nanomolar scale. Trends Genetics, 15(2):65-69, 1999.

[19] A. McIver and C. Morgan. Abstraction, Refinement And Proof For Probabilistic Systems (Monographs in Computer Science). SpringerVerlag, 2004.

[20] D. Monniaux. Abstract interpretation of probabilistic semantics. In Static Analysis Symposium, volume 1824 of Lecture Notes in Computer Science, pages 322-339. Springer, 2000.

[21] D. Monniaux. Backwards abstract interpretation of probabilistic programs. In Programming Languages and Systems, pages 367-382. Springer, 2001. 
[22] D. Monniaux. Abstract interpretation of programs as markov decision processes. Science of Computer Programming, 58(1):179-205, 2005.

[23] R. Motwani and P. Raghavan. Randomized Algorithms. Cambridge University Press, 1995.

[24] S. Sankaranarayanan, A. Chakarov, and S. Gulwani. Static analysis for probabilistic programs: inferring whole program properties from finitely many paths. In PLDI, pages 447-458. ACM, 2013.

[25] S. Sankaranarayanan, H. B. Sipma, and Z. Manna. Constraint-based linear-relations analysis. In Static Analysis Symposium (SAS 2004), volume 3148 of LNCS, pages 53-69. Springer, August 2004.

[26] D. Williams. Probability with Martingales. Cambridge University Press, 1991. 


\section{Probabilistic Loop Benchmarks}

\subsection{Example of Fixpoint Computation for PWL Loops}

In this section we present the application of the expectation invariant analysis to the concrete program in Example 1.1. We start with the largest possible cone of linear program expressions whose initial expectation $\mathbb{E}(\mathbf{e} \mid 0) \geq 0$. Our goal will be to refine this cone of candidate expressions into a cone of expectation invariant expressions.

Initially, we know the following facts: $\mathbb{E}(\mathrm{x} \mid 0)=-1, \mathbb{E}(\mathrm{y} \mid 0)=1, \mathbb{E}($ count $\mid 0)=0$. The set of expectation invariant candidate expressions (in PPL format) is then $E=\{1, \mathrm{x}+1,-\mathrm{x}-1, \mathrm{y}-1,1-$ $\mathrm{y}$, count, - count $\}$ since $\mathbb{E}(\mathrm{e} \mid 0) \geq 0$ hold for all e $\in E$. Therefore, $I_{0} \equiv C o n e(E)$ is a finitely generated cone of program expressions with non-negative expectations at step 0.

Next, consider the pre-expectation of $\mathbf{e}: c_{0}+c_{1} x_{1}+\cdots+c_{m} x_{m}$ under both transitions:

$$
\begin{aligned}
\operatorname{pre} \mathbb{E}_{\tau_{1}}(\mathbf{e}) & =c_{0}+c_{1}(\mathrm{x}+0.75)+c_{2}(\mathrm{y}+1.5)+c_{3}(\text { count }+1) \\
& =c_{0}+0.75 c_{1}+1.5 c_{2}+c_{3}+c_{1} \mathrm{x}+c_{2} \mathrm{y}+c_{3} \text { count } \\
\text { pre } \mathbb{E}_{\tau_{2}}(\mathbf{e}) & =c_{0}+c_{1} \mathrm{x}+c_{2} \mathrm{y}+c_{3} \text { count }
\end{aligned}
$$

Now $\mathcal{G}\left(I_{0}\right)=I_{0} \cap \operatorname{pre} \mathbb{E}\left(I_{0}\right)$ :

$$
\left(\exists \bar{\lambda}^{T} \geq 0\right)\left(\forall \tau_{i} \in \mathcal{T}\right)\left(\exists \mu_{i} \geq 0\right) \varphi(\mathbf{c}): \operatorname{pre} \mathbb{E}_{\tau_{i}}(\mathbf{e})=\bar{\lambda}^{T} \mathbf{E}+\mu_{i}^{T} \mathbf{g}_{\mathbf{i}}
$$

Constraints $\varphi_{0}(\mathbf{c})$ are called dual (in the linear programming sense) because they constrain the coefficients of the program expressions.

\begin{tabular}{|c|c||c|c|}
\hline & $\operatorname{pre} \mathbb{E}_{\tau_{1}}(\mathbf{e}):$ & & $\operatorname{pre} \mathbb{E}_{\tau_{2}}(\mathbf{e}):$ \\
\hline$(1)$ & $c_{0}+0.75 c_{1}+1.5 c_{2}+c_{3}=\lambda_{0}+\lambda_{1}-\lambda_{2}-\lambda_{3}+\lambda_{4}+10 \mu_{1}$ & $(5)$ & $c_{0}=\lambda_{0}+\lambda_{1}-\lambda_{2}-\lambda_{3}+\lambda_{4}-10 \mu_{2}$ \\
\hline$(2)$ & $c_{1}=-\lambda_{1}+\lambda_{2}-\mu_{1}$ & $(6)$ & $c_{1}=-\lambda_{1}+\lambda_{2}+\mu_{2}$ \\
\hline$(3)$ & $c_{2}=\lambda_{3}-\lambda_{4}-\mu_{1}$ & $(7)$ & $c_{2}=\lambda_{3}-\lambda_{4}+\mu_{2}$ \\
\hline$(4)$ & $c_{3}=\lambda_{5}-\lambda_{6}$ & $(8)$ & $c_{3}=\lambda_{5}-\lambda_{6}$ \\
\hline
\end{tabular}

These constraints are clearly satisfiable (even trivially satisfiable) and after performing projection we arrive at the following set of constraints: $\varphi_{0}(\mathbf{c}): 6 c_{0}-9 c_{1}-4 c_{3} \geq 0 \wedge 3 c_{1}+6 c_{2}+4 c_{3}=0$. The cone described by $\varphi_{0}(\mathbf{c})$ is precisely pre $\mathbb{E}\left(I_{0}\right)$. Conjoining $\varphi_{0}(c)$ to the constraints of $I_{0}$, therefore, amounts to computing $I_{1} \equiv \mathcal{G}\left(I_{0}\right)=I_{0} \cap \operatorname{pre} \mathbb{E}\left(I_{0}\right)$. In other words, conjoining the constraints removes from the cone

\begin{tabular}{|c|c|c|}
\hline Cone & Generators & Constraints \\
\hline$I_{0}$ & $\begin{array}{c}1, \mathrm{x}+1,-\mathrm{x}-1, \mathrm{y}-1 \\
1-\mathrm{y}, \text { count, }- \text { count }\end{array}$ & $c_{0}-c_{1}+c_{2} \geq 0$ \\
\hline$I_{1}$ & $\begin{array}{l}1,2-2 \mathrm{y}+3 \mathrm{count}, 4+4 \mathrm{x}-3 \mathrm{count} \\
-2+2 \mathrm{y}-3 \mathrm{count},-4-4 \mathrm{x}+3 \mathrm{count}\end{array}$ & $\begin{array}{c}3 c_{1}+6 c_{2}+4 c_{3}=0, c_{0}-c_{1}+c_{2} \geq 0 \\
6 c_{0}-9 c_{1}-4 c_{3} \geq 0\end{array}$ \\
\hline$I_{2}$ & $\begin{array}{l}1,2-2 \mathrm{y}+3 \text { count, } 4+4 \mathrm{x}-3 \mathrm{count} \\
-2+2 \mathrm{y}-3 \text { count },-4-4 \mathrm{x}+3 \mathrm{count}\end{array}$ & $\begin{array}{c}3 c_{1}+6 c_{2}+4 c_{3}=0, c_{0}-c_{1}+c_{2} \geq 0 \\
6 c_{0}-9 c_{1}-4 c_{3} \geq 0\end{array}$ \\
\hline
\end{tabular}
$I_{0}$ those expressions whose single step pre-expectation (even across one transition) does not belong to $I_{0}$.

The process is repeated where $\mathbf{E}$ is the generators of $I_{1}$. The reader is welcome to verify that $I_{2} \equiv$ $\mathcal{G}\left(I_{1}\right)=I_{0} \cap \operatorname{pre} \mathbb{E}\left(I_{1}\right)=I_{1}$. Therefore, $I^{*}=I_{2}$ is the fixpoint.

Finally, confirm that every expression in $I^{*}$ is expectation invariant by observing that $2-2 \mathrm{y}+3$ count and $4+4 \mathrm{x}-3$ count are martingales (Cf. Section 3.2). Therefore, every conic combination of generators in $I^{*}$ is also expectation invariant.

\section{2 mot-ex-loop-inv and mot-ex-poly}

Example 6.1. Consider the program in Example 3.2. Figure 3 presents the same program annotated with addition loop invariants $\varphi_{\text {l_inv }}$ obtained by running a traditional abstract interpretation analysis and 


\begin{tabular}{|c|c|c|}
\hline \multirow{2}{*}{ Variety } & \multicolumn{2}{|c|}{ Fixed Point } \\
\hline & Generators & Constraints \\
\hline MOT-EX & $\begin{array}{c}\mathrm{y}-2 \mathrm{x}, 3+2 \mathrm{x}-\mathrm{y},-3-2 \mathrm{x}+\mathrm{y} \\
4 \mathrm{x}+4 \mathrm{y}-9 \text { count },-4 \mathrm{x}-4 \mathrm{y}+9 \mathrm{count}\end{array}$ & $\begin{array}{c}3 c_{1}+6 c_{2}+4 c_{3}=0 \\
c_{0}-c_{1}+c_{2} \geq 0\end{array}$ \\
\hline MOT-EX-LOOP-INV & $\begin{array}{c}\text { count, } 56-9 \text { count } \\
-2+2 \mathrm{y}-3 \text { count, } 2-2 \mathrm{y}+3 \mathrm{count} \\
-4-4 \mathrm{x}+3 \mathrm{count}, 4+4 \mathrm{x}-3 \mathrm{count}\end{array}$ & $\begin{array}{c}9 c_{0}+33 c_{1}+93 c_{2}+56 c_{3} \geq 0 \\
c_{0}-c_{1}+c_{2} \geq 0\end{array}$ \\
\hline MOT-EX-POLY & $\begin{array}{c}4 \mathrm{x}^{2}-4 \mathrm{xy}+\mathrm{y}^{2}, 207 \mathrm{x}+89 \mathrm{x}^{2}-98 \mathrm{xy}+20 \mathrm{y}^{2}, \\
36 \mathrm{x}-27 \text { count }+16 \mathrm{x}^{2}-16 \mathrm{xy}+4 \mathrm{y}^{2}, \\
-36 \mathrm{x}+27 \text { count }-16 \mathrm{x}^{2}+16 \mathrm{xy}-4 \mathrm{y}^{2}, \\
6 \mathrm{x}-3 \mathrm{y}+4 \mathrm{x}^{2}-4 \mathrm{xy}+\mathrm{y}^{2}, \\
-6 \mathrm{x}+3 \mathrm{y}-4 \mathrm{x}^{2}+4 \mathrm{xy}-\mathrm{y}^{2}, \\
36 \mathrm{x}-64 \mathrm{x}^{2}-80 \mathrm{xy}+20 \mathrm{y}^{2}+216 \mathrm{xcount}-81 \text { count }^{2}, \\
-36 \mathrm{x}+64 \mathrm{x}^{2}+80 \mathrm{xy}-20 \mathrm{y}^{2}-216 \mathrm{xcount}+81 \text { count }^{2}, \\
9-4 \mathrm{x}^{2}+4 \mathrm{xy}-\mathrm{y}^{2}, \\
-9+4 \mathrm{x}^{2}-4 \mathrm{xy}+\mathrm{y}^{2}, \\
8 \mathrm{x}^{2}+4 \mathrm{xy}-4 \mathrm{y}^{2}-18 \mathrm{xcount}+9 \mathrm{ycount}, \\
-8 \mathrm{x}^{2}-4 \mathrm{xy}+4 \mathrm{y}^{2}+18 \mathrm{xcount}-9 \mathrm{ycount} \\
\end{array}$ & $\begin{array}{c}c_{0}-c_{1}+c_{2}+c_{4}-c_{5}+c_{6} \geq 0, \\
12 c_{1}+24 c_{2}+16 c_{3}+3 c_{4}+6 c_{5}+12 c_{6} \geq 0, \\
12 c_{1}+24 c_{2}+16 c_{3}+270 c_{4}+273 c_{5}+12 c_{6}+178 c_{7}=0 \\
24 c_{1}+48 c_{2}+32 c_{3}+6 c_{4}+279 c_{5}+1092 c_{6}+356 c_{8}=0 \\
27 c_{1}+54 c_{2}+36 c_{3}+207 c_{4}+414 c_{5}+828 c_{6}-356 c_{9}=0\end{array}$ \\
\hline
\end{tabular}

The interpretation of these results is as follows: for any generator expression e inside the fixed point, $(\forall n \geq 0) \mathbb{E}(\mathrm{e} \mid n) \geq 0$.

Finally, MOT-EX-POLY represents the original motivating program of Example 1.1 where we force our analysis to track all monomials over the program variables upto degree 2 . This is done by introducing a fresh variable for each monomial. Using this approach we were able to show interesting polynomial inductive expectation invariants. For example, when we combine generator expressions 5 and 6 , we obtain the following fact: $\mathbb{E}\left((2 x-y)^{2} \mid n\right)=\mathbb{E}(3(2 x-y) \mid n)$ for all $n \geq 0$. This fact could be use to reduce the complexity of a quadratic expression to a linear one in a larger static analysis framework.

\subsection{Other Benchmark Examples}

\subsubsection{2d-walk}

Example 6.2. The program in Figure 4 simulates a peg performing an infinite random walk in 2 dimensional space. The peg starts at the origin $(0,0)$ and first picks uniformly at random a direction $\{1: E ; 2: N ; 3: W ; 4: S\}$ and a uniform random displacement $r_{1} \sim$ unifRand(0,2). Depending on which quadrant the peg is in, the values of the $(x, y)$ cooradinates and distance from origin are updated accurately.

Our analysis was able to show the following IEI: $\mathbb{E}($ count $\mid n) \geq 0, \mathbb{E}(\mathrm{x} \mid n)=0, \mathbb{E}(\mathrm{y} \mid n)=0$, and $\mathbb{E}($ dist $\mid n)=0$, for all $n \geq 0$. This confirms the intuition and theory on random walks that in expectation the peg does not drift, in neither direction nor total distance, far away the origin.

\subsection{2 aggregate-rv}

Example 6.3. The program in Figure 5 simulates the aggregation of 500 independent identically distributed draws from a uniform random distribution over the interval [0,1]. Our analysis was able to generate the following inductive expectation invariants: $\mathbb{E}(\mathrm{N} \mid n)=500, \mathbb{E}(\mathrm{i}-2 \mathrm{x} \mid n)=0$, for all $n \geq 0$. Combining the IEI $\mathrm{i}-2 \mathrm{x}$ with the additional loop invariant $\mathrm{i} \leq \mathrm{N}$ we arrive at the $\mathbb{E}(\mathrm{x})=250$ at the end of the loop.

\subsection{3 hare-turtle}

Example 6.4. The program in Figure 6 models a stochastic version of Aesop's fable about the turtle and the hare. In this setting the hare chooses to wait until the turtle gains some significant advantage $(t=30$ initially) before starting to ponder whether to dash. At each time step the turtle proceeds at unit pace, whereas the hare first chooses uniformly at random whether to dash at this time instance. If he chooses to dash, his paces is a uniform draw over $[0,10]$. 


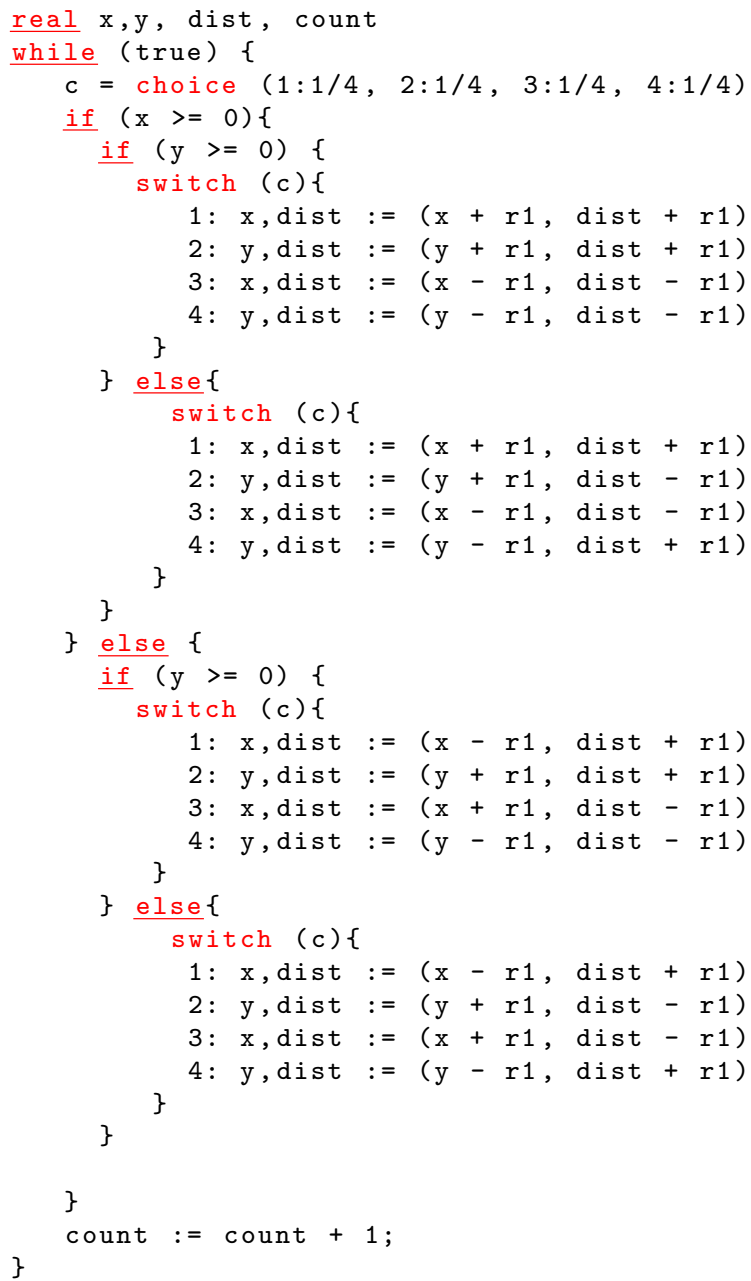

Figure 4: 2D-RANDOM-WALK (Left) Program modeling a peg on a random walk in 2D. (Right) The corresponding PWL probabilistic loop.

Given this setup our analysis was able to show the following IEI: $\mathbb{E}(5 \mathrm{t}-2 \mathrm{~h} \mid n) \geq 0, \mathbb{E}(2 \mathrm{~h}-5 \mathrm{t}+50 \mid n)=$ 0 , for all $n \geq 0$. This translates that in expectation (over the hare's choice to dash or not and hare's pace at each instant), the turtle is no more than 2.5 times slower than the hare. Additionally, $2 \mathrm{~h}-5 \mathrm{t}+50$ is a quantity that is conserved in expectation across any number $n \geq 0$ of iterations of the loop.

\subsection{4 coupon5}

Example 6.5. The program in Figure 7illustrates the Coupon Collector's Problem: Given $n=5$ distinct coupons, by choosing (with replacement) uniformly at random a coupon, what is the expected number of coupons (count) you should draw before collecting all $n=5$ coupons $(i=n)$. This is a well known problem where the expected number of coupons to draw grows as $\Theta(n \log n)$ in the number of coupons $n$.

For a linearized version of the problem, our analysis showed the following inductive expectation invariants: $\mathbb{E}(1.111 \mathrm{i}-$ count $\mid n) \geq 0, \mathbb{E}($ count $-\mathrm{i} \mid n) \geq 0, \mathbb{E}(4$ count $-5 \mathrm{i}+1 \mid n)=0$, for all $n \geq 0$. 
$\underline{\text { real }} x=0$;

real $N=500$;

for $(i=0 ; i<N ;++i)$

$\mathrm{x}=\mathrm{x}+$ unifRand $(0,1)$

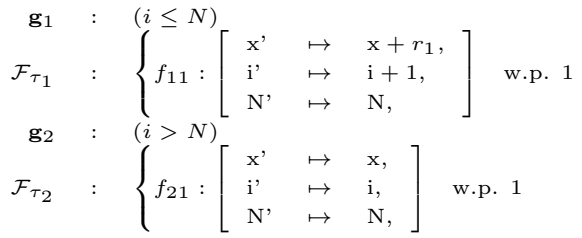

Figure 5: AGgREgate-RV (Left) Probabilistic program that accumulates 500 draws from a uniform random distribution. (Right) The corresponding PWL probabilistic loop.

real $\mathrm{h}, \mathrm{t}$;

$/ / \mathrm{h}$ is hare and $\mathrm{t}$ is tortoise $\mathrm{h}=0 ; \mathrm{t}=30$;

while $(\mathrm{h}<=\mathrm{t})\{$

if (flip (0.5) )

$\mathrm{h}=\mathrm{h}+$ unifRand $(0,10)$;

$t=t+1$

Figure 6: HARE-TURTLE (Left) Probabilistic program that models a stochastic version of the hare vs. turtle fable. $\mathrm{h}$ is whimsical in deciding when to move but quick; $\mathrm{t}$ is slow but consistent. (Right) The corresponding PWL probabilistic loop.

\subsection{5 hawk-dove}

Example 6.6. Figure 8 shows a model of a simple 2-player stochastic evolutionary game. In this game each player chooses one of two possible strategies: a collaborative strategy Dove or an aggressor strategy Hawk. If both players collaborate, they are guaranteed to split resources equally. In this setting Hawk is a strictly dominant strategy, which guarantees full amount of resource for the player in case opponent plays Dove. If both players attempt to use Hawk strategy then the outcome is stochastic: one player gets all resources while the other suffers penalty (injury).

At every round of the game the players carefully weight the penalty for losing a fight c against the common resources $\mathrm{v}$ for the round and perform a stochastic choice for a strategy ( player $_{i}$ ). Next, both strategies are evaluated and the corresponding balance of resources (pibal) is updated.

In the case of a fair balance of penalty to common resource ration (in HAWK-DOVE-FAIR), our analysis generates the following inductive expectation invariants: $\mathbb{E}($ count $\mid n) \geq 0, \mathbb{E}($ count - p1bal $\mid n)=0$, $\mathbb{E}($ count - p2bal $\mid n)=0, \mathbb{E}($ p2gain $\mid n)=0$, for all $n \geq 0$. The last IEI shows we can detect that the probabilistic choice of strategy as stated is indeed an evolutionary (stochastically) stable strategy that is also fair (no gain over other player). This last IEI cannot be established when the balance of $\mathrm{c}$ to $\mathrm{v}$ is broken (demonstrated in HAWK-DOVE-BIAS). 


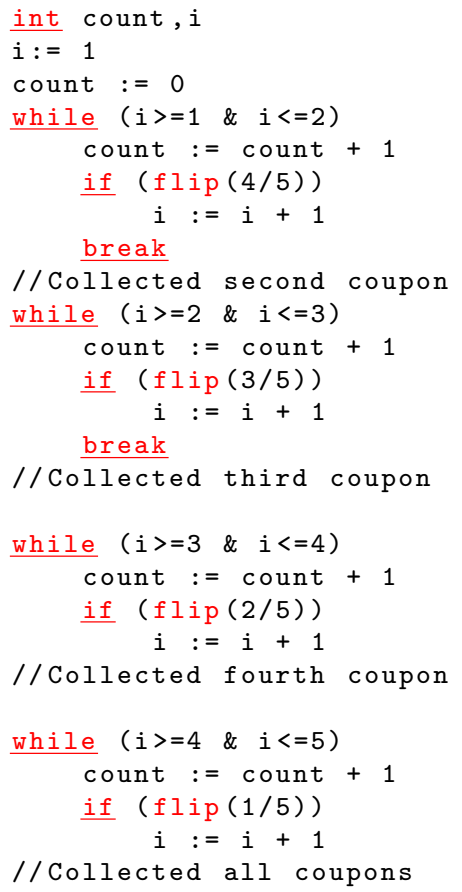

Figure 7: Coupon5 (Left) Probabilistic program that models the Coupon Collector Problem for $n=5$ coupons. (Right) The corresponding PWL probabilistic loop. 

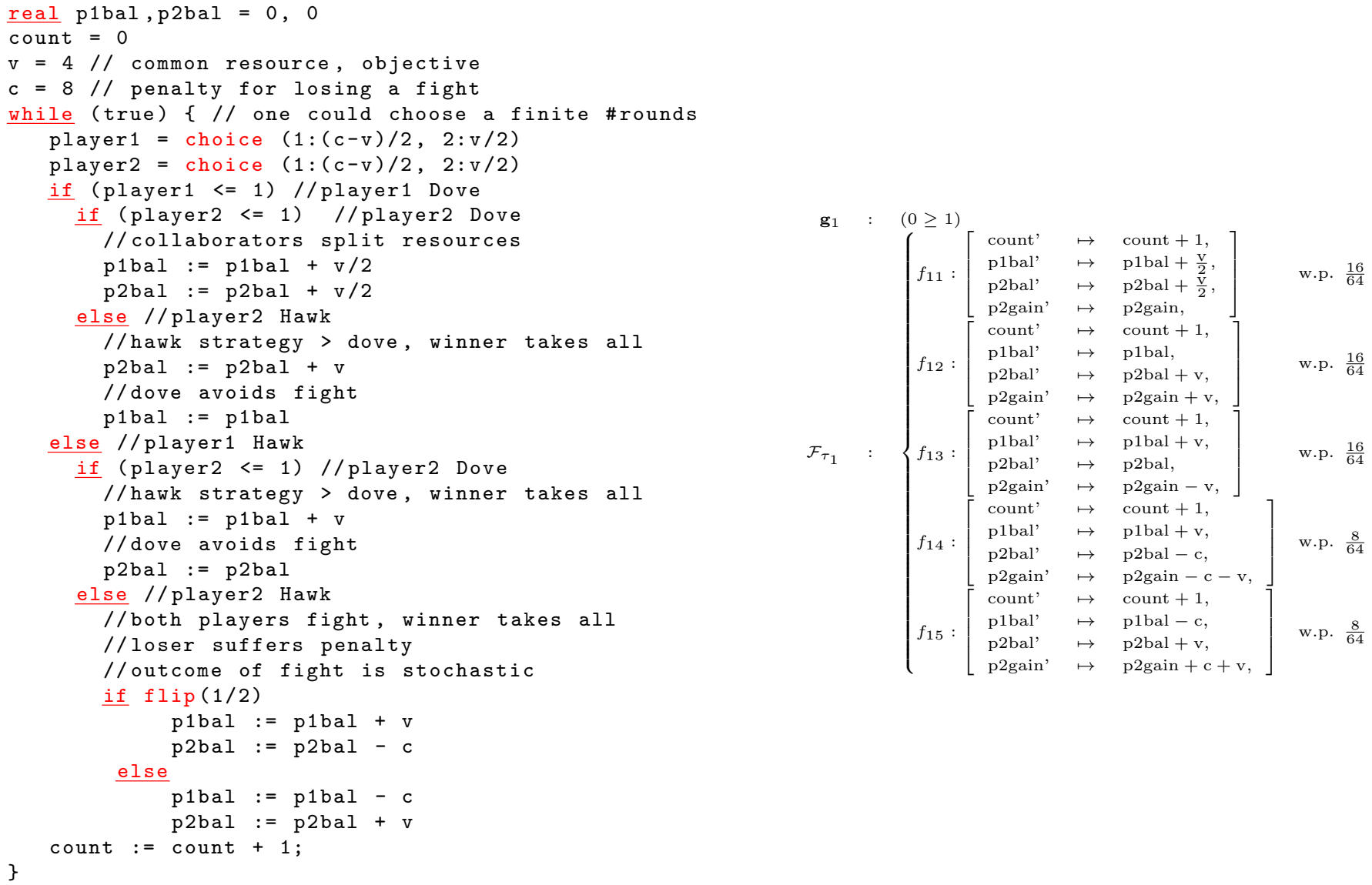

Figure 8: HAWK-DOVE (Left) Probabilistic program that models a stochastic 2-player evolutionary game of Dove and Hawk. (Right) The corresponding PWL probabilistic loop. 